Kinetic improvement of an algal diacylglycerol acyltransferase 1 via fusion with an acylCoA binding protein

4 Yang Xu ${ }^{\mathrm{a}}$, Kristian Mark P. Caldo ${ }^{\mathrm{a}}$, Lucas Falarz ${ }^{\mathrm{a}, \mathrm{b}}$, Kethmi Jayawardhane ${ }^{\mathrm{a}}$, Guanqun Chen ${ }^{\mathrm{a},{ }^{*}}$

$6{ }^{a}$ Department of Agricultural, Food and Nutritional Science, University of Alberta, Edmonton,

7 Alberta, Canada T6G 2P5

$8 \quad{ }^{\mathrm{b}}$ Department of Biological Sciences, University of Manitoba, Winnipeg, Manitoba, Canada R3T $9 \quad 2 \mathrm{~N} 2$

11 *To whom correspondence should be addressed: Guanqun Chen [Phone: (+1) 780 492-3148;

12 Fax: (+1) 780 492-4265; Email: gc24@ualberta.ca; ORCID: 0000-0001-5790-3903].

\title{
15 ABBREVIATIONS
}

16 ACBP, Acyl-CoA binding protein; C16:0, Palmitic acid; C18:0, Stearic acid; C18:1, Oleic acid;

17 C18:2, Linoleic acid; C18:3, $\alpha$-linolenic acid; DGAT, Diacylglycerol acyltransferase; ER,

18 Endoplasmic reticulum; GPAT, Glycerol-3-phosphate acyltransferase; OD 600 , Optical density at

$19600 \mathrm{~nm}$; S.D., Standard derivation; Sc-Ura, Synthetic complete medium lacking uracil; TAG,

20 Triacylglycerol; TLC, Thin-layer chromatography; TMD, Transmembrane domain; WRI,

21 Wrinkled. 


\section{SUMMARY}

23 Microalgal oils in the form of triacylglycerols (TAGs) are broadly used as nutritional 24 supplements and biofuels. Diacylglycerol acyltransferase (DGAT) catalyzes the final step of 25 acyl-CoA-dependent biosynthesis of TAG and is considered a key target for manipulating oil 26 production. Although a growing number of DGAT1s have been identified and over-expressed in 27 some algal species, the detailed structure-function relationship, as well as the improvement of 28 DGAT1 performance via protein engineering, remain largely untapped. Here, we explored the 29 structure-function features of the hydrophilic N-terminal domain of DGAT1 from the green 30 microalga Chromochloris zofingiensis (CzDGAT1). The results indicated that the N-terminal 31 domain of CzDGAT1 was less disordered than those of the higher eukaryotic enzymes and its 32 partial truncation or complete removal could substantially decrease enzyme activity, suggesting 33 its possible role in maintaining enzyme performance. Although the N-terminal domains of 34 animal and plant DGAT1s were previously found to bind acyl-CoAs, replacement of CzDGAT1 35 N-terminus by an acyl-CoA binding protein (ACBP) could not restore enzyme activity. 36 Interestingly, the fusion of ACBP to the N-terminus of the full-length CzDGAT1 could enhance

37 the enzyme affinity for acyl-CoAs and augment protein accumulation levels, which ultimately 38 drove oil accumulation in yeast cells and tobacco leaves to higher levels than the full-length 39 CzDGAT1. Overall, our findings unravel the distinct features of the N-terminus of algal DGAT1 40 and provide a strategy to engineer enhanced performance in DGAT1 via protein fusion, which 41 may open a vista in generating improved membrane-bound acyl-CoA-dependent enzymes and 42 boosting oil biosynthesis in plants and oleaginous microorganisms.

44 KEYWORDS: Triacylglycerol biosynthesis, DGAT, acyl-CoA binding protein, algal lipid, 45 enzyme kinetics, tobacco, yeast, Chromochloris zofingiensis 


\section{SIGNIFICANCE STATEMENT}

48 Here, we explored the N-terminus of a microalgal DGAT1, a membrane-bound enzyme

49 determining oil biosynthesis, using in silico analysis, truncation mutagenesis, protein fusion and

50 in vitro and in vivo characterization, and demonstrated its distinct structure-function features

51 from the higher eukaryotic enzymes. We further engineered enhanced performance in DGAT1

52 via N-terminal fusion of ACBP, and obtained a kinetically improved enzyme with augmented

53 protein production levels, which could boost oil accumulation in yeast and plant vegetative

54 tissues. 


\section{INTRODUCTION}

56 Plant-derived triacylglycerol (TAG) is one of the most abundant forms of energy storage and reduced carbon in nature, which has been widely used as food, feed and renewable feedstocks for industrial applications (Xu, Caldo, et al., 2018). Microalgae hold the promise of a sustainable bioresource of TAG because of the high ability to accumulate lipids and less competition for arable land with food crops (Xu, Caldo, et al., 2018; Hu et al., 2008). In recent years, the emerging research interest in exploring the oil biosynthesis mechanisms in microalgae has opened an important vista to fulfil the potential of microalgal oil production via physiological and genetic manipulations.

In microalgae, TAG assembly typically occurs through acyl-CoA-dependent and acylCoA-independent pathways by a series of acyltransferases which are universally present in plants and animals (Xu, Caldo, et al., 2018; Kong et al., 2018). Among them, acyl-CoA:diacylglycerol acyltransferase (DGAT, EC 2.3.1.20) catalyzes the acylation of $s n$-1,2-diacylglycerol with acylCoA to produce TAG, which is the final committed step in the acyl-CoA-dependent TAG biosynthesis. DGAT appears to play a prominent role in affecting the flux of carbon into TAG in many oilseed crops (Liu et al., 2012; Katavic et al., 1995; Zou et al., 1999; Weselake et al., 2008) and has been regarded as an important target for manipulation. Two major forms of membranebound non-homologous DGAT, designated DGAT1 and DGAT2, are known to predominantly contribute to TAG formation in developing seeds and microalgae. In plants, DGAT1 is considered as a major player in seed oil accumulation in some oil crops, such as rapeseed (Brassica napus) and safflower (Carthamus tinctorius) (Rahman et al., 2013; Tzen et al., 1993; Weselake et al., 1993), whereas DGAT2 appears to play a minor role in affecting oil production in oil crops. DGAT2, however, is important for incorporating unusual fatty acids into storage TAG in plants, such as tung tree (Vernicia fordii), castor (Ricinus communis), and ironweed (Vernonia galamensis) (Shockey et al., 2006; Kroon et al., 2006; Li et al., 2010). In microalgae, on the other hand, one or two copies of DGAT1 and several copies of DGAT2 were found to

81 likely contribute to the complexity of TAG formation, although their physiological roles remain

82 ambiguous (Mao et al., 2019; Chen and Smith, 2012; Gong et al., 2013; Liu and Benning, 2013;

$83 \mathrm{Xu}, \mathrm{Caldo}$, et al., 2018; Turchetto-Zolet et al., 2011; Liu et al., 2016). Given the importance of

84 the enzyme in governing the flux of substrates into TAG, over-expression of DGAT cDNAs have 85 been used to manipulate oil production in the seeds of Arabidopsis thaliana and oilseed crops 
such as soybean (Glycine max), B. napus, corn (Zea mays) and Camelina sativa (Jako et al., 2001; Weselake et al., 2008; Roesler et al., 2016; Kim et al., 2016; Oakes et al., 2011; Lardizabal et al., 2008; Li et al., 2012), in the leaves of Nicotiana tabacum, N. benthamiana, and Z. mays (Alameldin et al., 2017; Bouvier-Navé et al., 2000; Vanhercke et al., 2017; Chen et al., 2017), and in oleaginous yeast (Greer et al., 2015; Chen et al., 2017) and several microalgae including Chlamydomonas reinhardtii, Phaeodactylum tricornutum, and Nannochloropsis oceanica (Zulu et al., 2017; Xin et al., 2017; Xin et al., 2018; Mao et al., 2019; Iwai et al., 2014). the three-dimensional structure of which has not yet been elucidated (Xu, Caldo, et al., 2018). DGAT1 shares common features among different organisms, containing a very variable hydrophilic N-terminus with possibly distinct functions and a conserved C-terminal region with 8-10 predicted TMD (Liu et al., 2012). The hydrophilic N-termini of B. napus and mouse (Mus musculus) DGAT1s have been found to be involved in acyl-CoA binding and self-association (Weselake et al., 2006; Siloto et al., 2008; McFie et al., 2010). Recently, the structure of the Nterminal domain of B. napus DGAT1 was solved, revealing its important role as an enzyme regulatory domain that positively and negatively modulates enzyme activity (Caldo et al., 2017). The N-terminal domain of B. napus DGAT1 consists of two different segments, an intrinsically disordered region encompassing an autoinhibitory motif and a folded segment containing the allosteric site for acyl-CoA and CoA for activation and feedback inhibition of the enzyme,

105 respectively (Caldo et al., 2017). Although DGAT1s have been characterized from a growing 106 number of microalgal species (Kirchner et al., 2016; Wei et al., 2017; Guo et al., 2017;

107 Guihéneuf et al., 2011), the structure-function features of algal DGAT1, as well as using the 108 knowledge in improving DGAT performance, remain largely untapped.

111 carotenoid accumulation and industrial production, to explore the structure and function features 112 of the N-terminal domain of green microalgal DGAT1 and to investigate the potential of protein 113 fusion in improving DGAT1 performance. After comparing the evolutionary and structural

114 features of algal DGAT1 with the higher eukaryotic enzymes, the function of the hydrophilic N115 terminal domain of CzDGAT1 was examined via truncation mutagenesis, protein fusion and in 116 vitro enzyme assay. This $\mathrm{N}$-terminus was found to have very different features from those of the 
117 plant and animal enzymes and is important for maintaining high DGAT1 activity but not

118 essential for catalysis. The subsequent fusion of an A. thaliana acyl-CoA binding protein

119 (AtACBP6) to the N-terminus of CzDGAT1 resulted in a kinetically improved enzyme with

120 augmented protein production levels, and this improved DGAT1 variant could drive oil

121 accumulation to higher levels than the native DGAT1in yeast cells and $N$. benthamiana leaves.

122 The results indicated the fusion of ACBP with DGAT1 may represents a promising strategy in

123 engineering oil production in oleaginous organisms, which may also be used in engineering other

124 membrane-bound acyl-CoA-dependent enzymes.

\section{RESULTS}

\section{CzDGAT1 is phylogenetically related to plant DGAT1}

128 Phylogenetic analysis was carried out with DGAT1s from $C$. zofingiensis and other algae, plants

129 and animals. The two reported full-length $C z D G A T 1$ sequences, sharing $39.9 \%$ amino acid

130 pairwise identity, were both used in this analysis (Roth et al., 2017; Mao et al., 2019). As shown

131 in Figure 1, the results revealed some interesting features from an evolutionary perspective.

132 DGAT1s were found to be separated into four subgroups, with animal and plant DGAT1 falling

133 into two separate groups. DGAT1 from the charophyte green alga Klebsormidium nitens is

134 grouped with plant DGAT1, whereas CzDGAT1 and other DGAT1 from chlorophyte green

135 algae form a separate group, which is closely related to the plant DGAT1 group. Diatom DGAT1,

136 on the other hand, is clustered with fungal DGAT1 and is separate from all other sequences.

Further sequence analysis revealed that similar to plant and animal DGAT1s, the C-

138 terminal portion of algal DGAT1s contains 7-10 predicted transmembrane domains and is the

139 most conserved region, whereas the hydrophilic N-terminus preceding the first predicted

140 transmembrane domain is less conserved and variable in length (Figures 1 and S1 and Table S1).

141 Plant and animal DGAT1s have a hydrophilic N-terminus with a length of approximately 110

142 and 94 amino acid residues, respectively. On the contrary, the N-terminus of the algal DGAT1

143 has a quite variable length, ranging from 20 (Chlorella vulgaris DGAT1) to 326 amino acid

144 residues (Auxenochlorella protothecoides DGAT1). One CzDGAT1 isoform (isoform

145 CzDGAT1B in Mao et al., 2019) has a N-terminus composed of 107 amino acid residues (Table 
146

147

148

149

150

151

152

153

154

155

156

157

158

159

160

161

162

163

164

165

166

167

168

169

170

171

172

173

174

175

S1), which is similar to that of plant and animal DGAT1, and was used for subsequent experiments.

\section{CzDGAT1 encodes an active enzyme and has a hydrophilic $\mathbf{N}$-terminus with less} propensity to become disordered

The functionality of CzDGAT1 was characterized using the yeast mutant H1246, which is devoid of TAG biosynthesis ability (Sandager et al., 2002). The yeast complementation and fatty acid feeding assays showed that CzDGAT1 was able to restore TAG biosynthesis in

Saccharomyces cerevisiae mutant H1246 (Figure 2A) and facilitated the incorporation of the

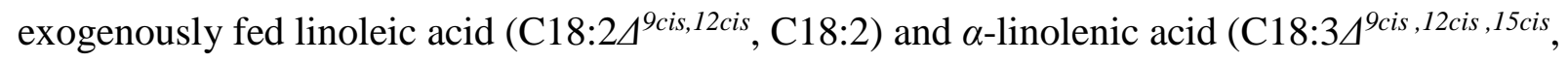
C18:3) into yeast TAG (Figure 2B). Further in vitro enzyme assay confirmed that CzDGAT1 displayed a strong DGAT activity (Figure 2C).

The N-terminal region of plant DGAT1 has been found to serve important regulatory functions (Caldo et al., 2017). The majority of the N-terminal hydrophilic region of plant and animal DGAT1 is likely present in a disordered state, whereas only a small portion preceding the first predicted transmembrane domain appears to have secondary structure (Figure 2D) (Caldo et al., 2017). To explore whether algal DGAT1 has similar features, the secondary structure of the N-terminal region of algal DGAT1 was analyzed using DISOPRED (Ward et al., 2004). Interestingly, CzDGAT1 may have a very different profile in the $\mathrm{N}$-terminus, where there is much less propensity to be disordered than that of B. napus DGAT1 (Figures 2E and F).

Similarly, N-terminus with less disordered state was also predicted in DGAT1 from a few other algal species (Figure S2). On the other hand, algal DGAT1 with an extremely long N-terminus, such as DGAT1 from K. nitens, is predicted to have both disordered and less disordered Nterminal segments. Furthermore, the folded portion of the N-terminal region of DGAT1 has been found to contain an allosteric site for binding of acyl-CoA and/or CoA has been identified in $B$. napus DGAT1 (Caldo et al., 2017). To test whether the N-terminus of algal DGAT1 also has the regulatory features, the N-terminal regions of DGAT1 from several plants, animal and algal species were aligned (Figure 2D). The four amino acid residues (R96, R97, R99 and E100 in $B$. napus DGAT1) implicated in CoA binding (Caldo et al., 2017) appear to be highly conserved in plant and animal DGAT1 but not in algal DGAT1, in which only the third residue (R99) is 
conserved. It should be noted that CzDGAT1 contains partial of the allosteric site in its Nterminus, where two out of the four amino acid residues involved in CoA binding are conserved (Figure 2D).

\section{N-terminal truncation of CzDGAT1 leads to less active enzymes}

Considering the partial conservation of the allosteric sites in the N-terminus of CzDGAT1, it is interesting to test whether the $\mathrm{N}$-terminus of CzDGAT1 also serves an analogous function to the B. napus DGAT1 N-terminus in catalysis. CzDGAT1 is predicted to have a 107-amino acid residue-long hydrophilic $\mathrm{N}$-terminal region, followed by 9 predicted hydrophobic segments (Figure 3A). To probe the possible role of the N-terminal region, the full-length CzDGAT1 and two N-terminal truncated versions were produced in S. cerevisiae mutant H1246 and the microsomal fractions containing the recombinant proteins were used to determine DGAT activity and protein production levels. Both the removal of the first 80 amino acid residues (CzDGAT1 $1_{81-}$ 550 ), which roughly correspond to the intrinsically disordered region in the $\mathrm{N}$-terminus of $B$. napus DGAT1 (Figure 2D), and the entire N-terminal region $\left(\mathrm{CzDGAT1}_{107-550}\right)$ led to reduced enzyme production levels (Figure 3B) and enzyme specific activities (Figure 3C). The specific activity of each enzyme was then normalized by the corresponding protein production level and the normalized activities of CzDGAT1 $1_{81-550}$ and CzDGAT1 $1_{107-550}$ were about 10 and 120-fold lower than that of the full-length enzyme, respectively (Figure 3D). These results suggest that the first 80 amino acids are not dispensable for the enzyme activity, and the entire N-terminal domain may be important for maintaining high enzyme activity.

\section{Fusion with ACBP at the N-terminus of CzDGAT1 and its N-terminal truncation mutant enhanced the enzyme production}

The acyl-CoA binding property of the N-terminal region has been revealed in B. napus DGAT1 (Weselake et al., 2006; Caldo et al., 2017). Considering the removal of the entire N-terminus of CzDGAT1 led to a nearly inactive enzyme, we further explored whether the $\mathrm{N}$-terminal fusion of CzDGAT1 107-550 with an ACBP would restore/improve the enzyme activity. We also tested if the fusion with ACBP could potentially improve DGAT performance by facilitating the channeling of acyl-CoA from the cytosol or the membrane lipid bilayer to the catalytic center of the enzyme. 
To test these hypotheses, AtACBP6, a 10-kDa soluble protein, was fused at the N-termini of CzDGAT1, CzDGAT1 $1_{81-550}$ and CzDGAT1 107-550, respectively, and the resulting fused proteins were produced in yeast $\mathrm{H} 1246$.

As shown in Figure 4A, N-terminal fusion with AtACBP6 largely increased the recombinant protein production in yeast, where the protein accumulation levels of AtACBP6

211 fused CzDGAT1, CzDGAT1 $1_{81-550}$ and CzDGAT1 107-550 were 5, 14, and 28-fold higher than the

212 corresponding unfused CzDGAT1 variants, respectively. The specific activities of the AtACBP6

213 fused CzDGAT1 and CzDGAT1 $1_{81-550}$ were also improved to about 2 and 5-fold greater than the

214 unfused enzymes, respectively (Figure 4B). N-terminal fusion of CzDGAT1 107-550 with

215 AtACBP6, however, led to no improvement in enzyme activity compared to CzDGAT1 107-550

216 (Figure 4B). The normalized enzyme activities of AtACBP6 fused CzDGAT1 and CzDGAT1 ${ }_{81-}$

217550 were about 2.5 and 3-fold lower than the unfused enzymes, respectively (Figure 4C),

218 suggesting that the enhanced enzyme activities of the fused proteins were mainly due to the

219 enhanced protein abundance.

\section{N-terminal fusion with ACBP kinetically improves CzDGAT1 and its N-terminal} 222 truncation mutant

223 To further explore the effects of fusing ACBP with DGAT1, the activities of the full-length

224 CzDGAT1, ACBP-fused full-length CzDGAT1, CzDGAT181-550, and ACBP-fused CzDGAT181-

225550 were analyzed over increasing concentrations of oleoyl-CoA (Figures 5A-D). The full-length

226 enzyme and the N-terminal truncation mutant had a similar response to the increasing acyl-donor

227 concentration, with the maximum enzyme activity achieved at 5 and $7.5 \mu \mathrm{M}$ oleoyl-CoA,

228 respectively. The ACBP-fused CzDGAT1 1-550 and CzDGAT181-550, on the other hand, reached

229 the maximum enzyme activity at lower concentrations at 3 and $5 \mu \mathrm{M}$ oleoyl-CoA, respectively.

230 The substrate saturation curves for CzDGAT1 1-550 and CzDGAT1 $181-550$ and their ACBP-fused

231 versions had better fits to the allosteric sigmoidal equation over the Michaelis-Menten equation

232 (Figures 5A-D). The Hill coefficients of CzDGAT1 1-550, ACBP-fused CzDGAT1, CzDGAT1 81 -

233550 and ACBP-fused CzDGAT1 $81-550$ were 1.39 $\pm 0.06,1.91 \pm 0.12,1.71 \pm 0.06$, and 1.57 \pm 0.14 ,

234 respectively, which indicates that all these enzymes exhibited positive cooperativity. In addition,

235 CzDGAT1 1-550 and CzDGAT1 $1_{1-550}$ had apparent $\mathrm{S}_{0.5}$ values of $1.48 \pm 0.07$ and $1.83 \pm 0.05 \mu \mathrm{M}$ 
oleoyl-CoA, respectively (Table 1), suggesting that the oleoyl-CoA affinity of CzDGAT1 ${ }_{81-550}$ might be lower than that of the full-length enzyme. Interestingly, ACBP-fused CzDGAT1 1-550 and ACBP-fused CzDGAT1 $1_{81-550}$ had apparent $S_{0.5}$ values of $0.94 \pm 0.04$ and $1.50 \pm 0.10 \mu \mathrm{M}$ oleoyl-CoA, respectively, which are 1.6 and 1.2-fold lower than the values of the corresponding unfused enzymes (Table 1). This result suggests that fusion with ACBP could enhance the affinity of DGAT variants to oleoyl-CoA.

N-terminal fusion with ACBP in CzDGAT1 boosts oil content in yeast and tobacco leaves

244 In order to determine whether the ACBP fused CzDGAT1 variants can boost oil production to higher levels compared to the native enzyme, cDNAs encoding the full-length CzDGAT1, the Nterminal truncated and the ACBP fused versions were individually introduced into the yeast strain H1246. Expression of $A C B P$ or $L a c Z$ and co-expression of $C z D G A T 1_{1-550}$ or $C z D G A T 1_{81 \text { - }}$ 550 with $A C B P$ in yeast were used as controls. The production of $\mathrm{CzDGAT} 1_{1-550}$ led to considerably increased neutral lipid accumulation in yeast at $24 \mathrm{~h}$ and $72 \mathrm{~h}$, whereas no change in neutral lipid production was observed for the yeast expressing $C_{z} D G A T 1_{81-550}$ when compared to the LacZ control (Figure 6A). Fusion with ACBP at the N-terminal of CzDGAT1 1-550 but not the N-terminal truncated enzyme was able to further increase the neutral lipid content, resulting in about 2-fold higher production than the native enzyme (Figure 6A). It should be noted that coexpression of $A C B P$ with $C z D G A T 1_{1-550}$ also promoted neutral lipid production in yeast to a comparable level to ACBP-fused CzDGAT11-550 (Figure 6A), suggesting that ACBP may improve the acyl-CoA availability to DGAT and thus enhance the lipid production. vegetative tissues, cDNAs encoding CzDGAT11-550, ACBP-fused CzDGAT11-550, CzDGAT181550 or ACBP-fused CzDGAT181-550 were transiently co-expressed with A. thaliana Wrinkled1 (AtWRII) in the $N$. benthamiana leaves, respectively. AtWRII encodes a transcription factor involved in the upregulation of genes in late glycolysis and fatty-acid biosynthesis (Xu, Caldo, et 262 al., 2018) and co-expression of AtWRII and DGAT1 was previously found to substantially 263 increase the TAG production in N. benthamiana leaves (Vanhercke et al., 2013). The over264 expression of a cDNA encoding CzDGAT1 1-550 or ACBP-fused CzDGAT1 1-550 and the co265 expression of $C z D G A T 1_{1-550}$ and $A C B P\left(\mathrm{ACBP}+\mathrm{DGAT}_{1-550}\right)$ led to considerable increases in 
leaf TAG content compared with the expression of AtWRI1 alone (Figure 6B). Interestingly, ACBP-fused CzDGAT1 1-550 had a higher impact on improving the leaf TAG production than CzDGAT1 1-550 or the co-expression group (ACBP + DGAT1 1-550), where a 1.40 or 1.35 -fold increase in TAG content was observed, respectively (Figure 6B). Consistently, the TAG fatty acid composition was also affected in the groups with increased TAG content compared with the AtWRII control group, in which palmitic acid (C16:0) and C18:3 contents were decreased while

272 the content of stearic acid (C18:0), oleic acid (C18:1 $\left.\Delta^{\text {cis9 }}, \mathrm{C} 18: 1\right)$, and C18:2 were increased

273 (Figure 6C). The over-expression of a cDNA encoding ACBP-fused CzDGAT1 ${ }_{81-550}$ also resulted in a slight increase in TAG production relative to that of the unfused enzyme, although

275 both groups showed no significant difference in TAG content and composition compared to the 276 AtWRII control group, with an exception of a decrease in C16:0 content in the ACBP-fused CzDGAT1 $1_{81-550}$ (Figures 6B and C).

To test whether ACBP fusion affects the subcellular localization of CzDGAT1, Venus, a variant of yellow fluorescent protein, was fused to the N-terminal of CzDGAT1 1-550 or ACBPCzDGAT1 1-550, and was transiently co-produced with $A$. thaliana glycerol-3-phosphate acyltransferase (AtGPAT9) containing a C-terminal SCFP3A (a cyan fluorescent protein) fusion in tobacco leaves, which is known to reside in the endoplasmic reticulum (ER) (Gidda et al., 2009). Both CzDGAT1 1-550 or ACBP-CzDGAT1 1-550 were found to co-localize with AtGPAT9

284 (Figure 6D), suggesting their ER localization. It should also be noted that linkage of ACBP to the 285 N-terminus of CzDGAT1 enhanced the CzDGAT1 production in tobacco leaves as indicated by 286 the increased fluorescence intensity (Figure 6E) and enhanced protein accumulation levels based 287 on Western blot analysis (Figures 6F and G).

\section{DISCUSSION}

290 Recently, DGAT1s from a growing number of microalgal species have been characterized with 291 the focus on elucidating their physiological role and application in manipulating oil production 292 (Kirchner et al., 2016; Wei et al., 2017; Guo et al., 2017; Guihéneuf et al., 2011). The structure293 function perspectives of algal DGAT1 and the relationship to plant and animal DGAT1, however, 294 remain largely unexplored. In the current study, the evolutionary and structural features of algal 295 DGAT1 in comparison to the higher eukaryotic enzymes were investigated via in silico analysis, 
truncation mutagenesis and in vitro enzyme assay, using DGAT1 of the emerging model green microalga C. zofingiensis (Roth et al., 2017; Mao et al., 2019; Liu et al., 2019) as a representative. Moreover, an improved DGAT1 variant was engineered by N-terminal fusion with ACBP, and its potential in enhancing oil production was explored using yeast expression and $N$. benthamiana transient expression systems.

The evolutionary and sequence analyses revealed that CzDGAT1 and other green microalgal DGAT1 are closely related to the plant DGAT1 clade. In addition, algal DGAT1 has similar structural features to plant and animal DGAT1, such as the highly conserved C-terminal hydrophobic region forming multiple TMDs and the variable hydrophilic N-terminal region (Figures 1 and S1 and Table S1). Interestingly, the N-terminus of algal DGAT1 varies dramatically in length ranging from 20 to 326 amino acid residues, which differs from that of plant and animal DGAT1 (Figure 1 and Table S1). The large variation in the N-terminal size appears to be not relevant to the maintenance of enzyme activity, since many DGAT1s with extremely long or short $\mathrm{N}$-termini have been demonstrated to function in TAG biosynthesis (Kirchner et al., 2016; Wei et al., 2017; Mao et al., 2019). Furthermore, the N-terminal region of algal DGAT1 is likely to have different structural features from that of plant and animal DGAT1. The majority of the N-terminus of plant and animal DGAT1 is disordered, while the small folded portion contains a conserved allosteric site (Caldo et al., 2017). On the contrary, the algal DGAT1 N-terminal region has much less propensity to be disordered based on secondary structure analysis (Figures 2E, F and S2) and the possible allosteric site is less conserved in algal DGAT1 (Figure 2D). Taken together, these results suggest that the N-terminal region of algal DGAT1 may have evolved differently from that of plant and animal in terms of structure and function.

The distinct features of the N-terminal domain of algal DGAT1 in enzyme catalysis are also supported by the evidence from the truncation mutagenesis of CzDGAT1. Previous study on

322 regulates enzyme activity and the removal of that region led to higher enzyme activity (Caldo et 323 al., 2017). CzDGAT1 has a N-terminal region (1-107) of a similar size to that of plant and 324 animal DGAT1 (Table S1), but truncation of the equivalent region to the autoinhibitory motif in 325 B. napus DGAT1 (CzDGAT181-550; Figure 2D) diminished the enzyme activity by 10-fold 
326 (Figure 3D), suggesting that this region may not function as an autoinhibitory motif in

327 CzDGAT1. Indeed, the DISOPRED analysis suggested that the N-terminal domain of CzDGAT1

328 does not have a strong tendency to have the disordered region where autoinhibitory motifs are

329 normally found (Figures $2 \mathrm{E}$ and F). Moreover, the N-terminal domain was found to be involved

330 in mediating positive cooperativity in B. napus DGAT1, where the N-terminal truncated $B$.

331 napus DGAT181-501 had a decreased Hill coefficient value compared to the full-length enzyme

332 (Caldo et al., 2017). This appears not to be the case for CzDGAT1 since both CzDGAT1 and

333 CzDGAT181-550 exhibited positive cooperativity with close Hill coefficient values (Figures 5A

334 and B, Table 1). Interestingly, N-terminal truncation of CzDGAT1 (CzDGAT181-550) seems to

335 alleviate the substrate inhibition of the full-length enzyme (Figure S3 and Table S2), suggesting

336 that segment 1-80 may be related to a low affinity non-catalytic acyl-CoA binding site.

337 Furthermore, the N-terminal domains of B. napus and mouse DGAT1 were shown to be

338 responsible for oligomer formation (McFie et al., 2010; Weselake et al., 2006; Caldo et al.,

339 2017). Although whether this domain functions in self-association in CzDGAT1 remains further

340 exploration, DISOPRED analysis showed that the N-terminus of CzDGAT1 has a less propensity

341 to form protein-protein interactions than B. napus and mouse DGAT1 (Figures 2E, F and S2). In

342 addition, truncation of this domain did not affect the self-interaction of CzDGAT1 in the

343 membrane yeast two-hybrid assay (Figure S4). It is also possible that the observed self-

344 interaction was due to the presence of a region in the hydrophobic part of CzDGAT1 that is

345 involved in oligomer formation. Indeed, a 16-kDa fragment of mouse DGAT1 present in the ER

346 lumen was also found to likely form dimer/tetramer (McFie et al., 2010).

The removal of the entire N-terminal region in CzDGAT1 (CzDGAT1 107-550) almost

348 inactivated the enzyme (Figure 3D), suggesting that the N-terminal region is not necessary for

349 enzyme catalysis but is important for maintaining high enzyme activity. This agrees with the

350 previous studies on the B. napus DGAT1 (Caldo et al., 2017). Previously, the N-termini of $B$.

351 napus and mouse DGAT1 were found to associate with acyl-CoA in a sigmoidal fashion

352 (Weselake et al., 2006; Siloto et al., 2008). Replacement of the N-terminal region by ACBP in

353 CzDGAT1 (ACBP fused CzDGAT1 107-550), however, was not able to restore or improve the

354 enzyme activity (Figure 4), suggesting that the $\mathrm{N}$-terminus may function as a regulatory domain

355 rather than as an acyl-CoA binding site. Indeed, the last 20-30 amino acid residues in the N-

356 terminus of B. napus DGAT1 has a well-folded structure and constitutes a regulatory domain for 
allosteric binding of acyl-CoA and CoA, where the binding of CoA would trigger the subsequent inhibition of DGAT activity (Caldo et al., 2017). The allosteric site for CoA, however, is only partially conserved in CzDGAT1 (Figure 2D), and the activity of CzDGAT1 was not affected in the presence of $50 \mu \mathrm{M}$ CoA (Figure S5), suggesting that the allosteric site for CoA might be not present in the algal DGAT1 or not activated under this experimental condition. The CoA inhibition was also not triggered by fusion with ACBP to CzDGAT1 (Figure S5), despite that ACBP is able to bind CoA alone (Robinson et al., 1996). However, we cannot rule out the possibility that failure in restoring DGAT activity by replacing the N-terminus with ACBP may be caused by the high binding affinity of ACBP to acyl-CoAs, while a moderate binding affinity being required for the enzyme function. A comprehensive characterization of the $\mathrm{N}$-termini of CzDGAT1 and other algal DGAT1s with respective to the acyl-CoA binding ability would be an interesting next step to explore the possible role of this domain in the catalysis of algal DGATs.

The two isoforms of DGAT1 from the green microalga $C$. zofingiensis (Mao et al., 2019) have very distinct features. They share $39.9 \%$ amino acid pairwise identity and have very different lengths mainly attributable to the variable N-terminal regions (Figure S1). The Nterminus of the CzDGAT1 isoform studied in the current study is of a similar length to that of plant and animal DGAT1 but with less tendency to be disordered, whereas the other isoform has an extremely long hydrophilic N-terminus of 289 amino acid residues with the first 160 amino acid residues showing low tendency to become disordered and the segment consisting of the remaining 129 residues being highly disordered (Table S1 and Figure S2). Since DGAT1 isoform with a longer N-terminus was found to have a higher ability to restore TAG biosynthesis in the yeast mutant (Mao et al., 2019), it is possible that their different $\mathrm{N}$-terminal domain features may directly impact on the enzyme activity and accumulation. Therefore, it would be interesting to comprehensively compare the structure and function features of the $\mathrm{N}$-terminal domains of both CzDGAT1 isoforms in the future. Additionally, it may also be worthwhile to explore the activities and physiological functions of CzDGAT1 in detail, especially considering that the coding genes of both isoforms were up-regulated under nitrogen deprivation and high-

384 light stress conditions to contribute to TAG accumulation but with very different transcript 385 abundances (under both conditions) and response patterns (under high-light stress) (Roth et al., 2017; Mao et al., 2019; Liu et al., 2019). 
It is interesting to note that N-terminal fusion with ACBP could markedly increase the yeast recombinant protein production up to 28 folds (Figure 4A). It has been suggested that the identity of the amino acid residues at the N-terminus of proteins could potentially affect the protein turnover and/or translation rate and thus enhance the protein production (Greer et al., 2015; Sriram et al., 2011). Indeed, the addition of N-terminal tag was found to increase the production of B. napus DGAT1 and plant fatty acid desaturases in yeast cells (O'Quin et al., 2009; Greer et al., 2015). Previously, fusion with small soluble proteins, such as thioredoxin, ubiquitin and maltose binding protein was also found to augment the accumulation levels of recombinant protein in Escherichia coli and/or yeast likely by improving protein stability and/or translation efficiency (Marsh et al., 1989; Pryor and Leiting, 1997; Jacquet et al., 1999; Ecker et $a l ., 1989)$. The protein fusion strategy, especially fusion to ubiquitin, has also met considerable success in increasing protein production in transgenic plants (Mishra et al., 2006; Tian and Sun, 2011; Hondred et al., 1999; Streatfield, 2007). Our finding that linkage to ACBP enhanced the CzDGAT1 production in tobacco leaves (Figures 6E-G) suggests that ACBP might also have potential to be used as a protein fusion partner to enhance protein accumulation in plants, especially for membrane-bound acyl-CoA-dependent enzymes.

Fusion with ACBP not only augmented the production of CzDGAT1 (Figures 4A and $6 \mathrm{E}-\mathrm{G}$ ) but also improved the kinetic parameters of the enzyme (Figure 5). Our kinetic analysis showed that ACBP-fused CzDGAT1 1-550 or ACBP-fused CzDGAT181-550 still exhibited positive cooperativity despite that ACBP binds acyl-CoA in a typical hyperbolic manner (Brown et al., 1998; Yurchenko et al., 2009) and, more importantly, the fused enzymes had increased affinity for oleoyl-CoA with the apparent $\mathrm{S}_{0.5}$ values decreasing by 1.6 and 1.2-fold, respectively (Table 1 and Figure 5). DGAT affinity for oleoyl-CoA has been reported as an important determinant of oil production since a strong correlation of oleoyl-CoA affinity with oil content was found in soybean expressing DGATl variants (Roesler et al., 2016). Cytosolic 10-kDa ACBP, consisting 412 of four $\alpha$ helixes (Figure S6), is capable of binding acyl-CoAs with high affinity (Robinson et al., 413 1996; Du et al., 2016). Considering the increased DGAT affinity for oleoyl-CoA of the ACBP414 fused CzDGAT1, we proposed that ACBP would facilitate the feeding of acyl-CoA to the 415 catalytic pocket of CzDGAT1 via capturing cytosolic acyl-CoAs (or those partitioned into the membrane lipid bilayer) and subsequent channeling to DGAT by proximity (Figure S6).

417 Similarly, fusion of proteins in a consecutive reaction has been shown to have synergistic effects 
418 in substrate conversion in a single catalytic region (Elleuche, 2015). ACBP has been implicated

419 in acyl-CoA binding and transport, which maintains the substrate supply for the acyl-CoA-

420 dependent acyltransferases in the ER, including DGAT (Yurchenko and Weselake, 2011).

421 Recently, ACBP2 was found to probably interact directly with lysophospholipase 2 in $A$.

422 thaliana and thereby facilitate the lysophosphatidylcholine hydrolysis (Miao et al., 2019).

423 Therefore, it is plausible to assume the presence of transient interactions between ACBP and

424 DGAT, the enhancement of which via protein fusion may further lead to an efficient substrate

425 channeling. Furthermore, B. napus ACBP has been shown to slightly stimulate DGAT1 activity

426 when the concentration of acyl-CoA is higher than that of ACBP, but to inhibit DGAT activity

427 when ACBP is in excess likely due to the competition of ACBP with ACBP-bound acyl-CoA or

428 DGAT for enzyme-substrate interaction (Yurchenko and Weselake, 2011). ACBP-fused

429 CzDGAT1 or CzDGAT1 181-550, on the other hand, showed a more rapid response to increasing

430 acyl-CoA concentration from 0.1 to 5 or $10 \mu \mathrm{M}$ than the unfused enzymes, respectively (Figure 5

431 and Table 1), suggesting that in the 1:1 fusion form ACBP may facilitate enzyme-substrate

432 binding rather than compete with DGAT for substrate.

The kinetically improved DGAT1 via fusion with ACBP shows promises in oil

434 production in both yeast and plant systems (Figure 6). Although ACBP-fused DGAT1 was able

435 to augment the yeast neutral lipid production up to two-fold relative to that of the unfused

436 enzyme, a comparable level was also achieved in the yeast co-expressing individual fusion

437 groups (Figure 6A). In tobacco transient expression system, ACBP-fused DGAT1 was more

438 efficient to boost leaf TAG content than the unfused enzyme and the co-expressed group. It

439 should be noted that the sizes of the acyl-CoA pool in yeast and tobacco leaf cells are different.

440 The cellular concentration of total acyl-CoA pool in S. cerevisiae has been reported to be in the

441 range of 10-42 $\mu \mathrm{M}$ depending on the strain and its metabolic state (Schjerling et al., 1996;

442 Mandrup et al., 1993; Knudsen et al., 1994); whereas the concentration of total acyl-CoAs in

443 tobacco leaves was determined to be $0.5 \mu \mathrm{M}$ (Moreno et al., 2014) [intracellular concentrations

444 were converted to $\mu \mathrm{M}$ assuming a specific cell volume of $3.7 \times 10^{-14} \mathrm{~L} /$ cell (Krink-Koutsoubelis

445 et al., 2018) and $1 \mathrm{mg}$ equal to $1 \mu \mathrm{L}$ volume (Larson and Graham, 2001), for yeast and plant cells,

446 respectively]. Co-production of ACBP and DGAT1 appears to work better in the cells with the

447 acyl-CoA concentration at higher levels rather than lower levels (Figures 6A and B). It is

448 possible that co-production of ACBP with DGAT1 affected in vivo DGAT1 activity in a manner 
dependent on the cellular acyl-CoA concentration, where co-produced ACBP stimulates DGAT1 activity at high acyl-CoA concentration but restricts DGAT1 activity at low acyl-CoA concentration, which agrees with the previous finding on the in vitro stimulation of DGAT activity by B. napus ACBP (Yurchenko and Weselake, 2011). ACBP-fused DGAT1, on the other

453 hand, showed good performance in cells with both low and high concentrations of acyl-CoAs

454 (Figures 6A and B) likely due to the improved enzyme kinetics and protein production levels, 455 despite that fusion with ACBP slightly enhanced the substrate inhibition of DGAT1 at the acyl-

456 CoA concentrations above $10 \mu \mathrm{M}$ (Figure S3 and Table S2). Moreover, it would be interesting to 457 explore the potential of co-producing DGAT with other ACBP isoforms, such as ER-bound 458 ACBP, in improving TAG production, since both soluble and ER-bound ACBP may be involved 459 in the capture and shuttle of acyl-CoAs for downstream acyl-CoA-dependent acyltransferases. In conclusion, our results suggested that algal DGAT1 may have different evolutionary and structural features from plant and animal DGAT1 with respect to the hydrophilic N-terminal domain. This domain is predicted to be present in a less disordered state in CzDGAT1 than that of animal/plant DGAT1. Although the N-terminal domain is not necessary for acyltransferase activity of CzDGAT1, its removal, however, led to huge decreases in CzDGAT1 enzyme activity, which cannot be restored by fusion with an ACBP. We also found that fusion of ACBP to the Nterminus of the full-length CzDGAT1 could not only augment the protein accumulation levels in yeast and tobacco leaves but also kinetically improve the enzyme. ACBP-fused DGAT1 was

468 more effective in improving the oil contents of yeast cells and vegetative tissues than the native DGAT1. This strategy may have great potential in engineering membrane-bound acyl-CoAdependent enzymes and manipulating oil biosynthesis in plants, algae and other oleaginous

471 organisms.

\section{EXPERIMENTAL PROCEDURES}

\section{Sequence analysis}

475 Multiple sequence alignments of DGAT1 proteins from different animal, plant and microalgal 476 species (Table S1) were performed using the L-INS-i method implemented in MAFFT v7.271

477 (Katoh and Standley, 2013) and trimmed using trimAl v1.2 (Capella-Gutiérrez et al., 2009). The 
resulting alignments were used for model selection using IQ-TREE (v1.3.11.1) with the option "m TESTONLY”. The phylogenetic tree was then constructed with the best-fit model for protein alignment (LG+F+I+G) using Phyml v3.0 (Guindon et al., 2010) and visualized with iTOL v3 (Letunic and Bork, 2016). The topology organization of DGAT1 was predicted using TMHMM (Krogh et al., 2001). variants

486 The coding sequence of $C z D G A T 1$ (Phytozome accession number: Cz09g08290) was chemically 487 synthesized (General Biosystems, Morrisville, NC), and re-cloned into the pYES2.1 yeast expression vector (Invitrogen, Burlington, Canada) under the control of the galactose-inducible GAL1 promoter. N-terminal truncation mutants of $C z D G A T 1$ were PCR-amplified and subcloned into the pYES2.1 vector. The coding sequence of AtACBP6 (NCBI accession number: NM_102916) was PCR-amplified from the plasmid pAT332 (kindly provided by Dr. Mee-Len

492 Chye, University of Hong Kong) (Chen et al., 2008), and subcloned into the pYES2.1 vector and the pESC-leu2d(empty) vector (a gift from Dr. Jay Keasling; Addgene plasmid \# 20120) (Ro et al., 2006). To generate ACBP-CzDGAT1 1-550, ACBP-CzDGAT1 $1_{81-550}$ and ACBP-CzDGAT1 ${ }_{107-}$ 550 fusion proteins, the coding sequences of $A t A C B P 6$ and variant $C z D G A T 1 s$ were individually amplified and the resulting amplicons were fused using overlap extension PCR. The DNA sequences of ACBP-CzDGAT1 1-550, ACBP-CzDGAT1 $181-550$ and ACBP-CzDGAT1 ${ }_{107-550}$ fusion proteins were then subcloned into the pYES2.1 vector. The stop codon was removed from each sequence for in-frame fusion with a C-terminal V5 tag, which is encoded in the pYES2.1 vector. The primers used for the preparation of all the constructs are listed in Table S3.

After the integrity of each construct was confirmed by sequencing, the constructs were

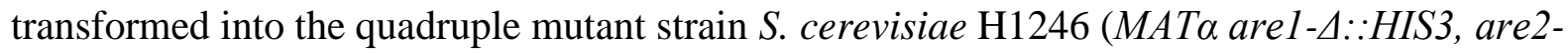

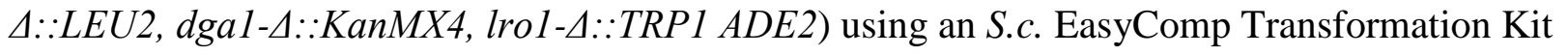

504 (Invitrogen) for yeast heterologous expression (Xu, Holic, et al., 2018; Xu et al., 2017; Xu et al., 505 2019). The recombinant yeast cells were first cultured overnight in liquid minimal medium containing $0.67 \%(\mathrm{w} / \mathrm{v})$ yeast nitrogen base, $0.2 \%(\mathrm{w} / \mathrm{v})$ synthetic complete medium lacking uracil (SC-Ura) and 2\% (w/v) raffinose. The yeast cultures were then used to inoculate the 
induction medium containing $0.67 \%(\mathrm{w} / \mathrm{v})$ yeast nitrogen base, $0.2 \%(\mathrm{w} / \mathrm{v}) \mathrm{SC}-\mathrm{Ura}, 2 \%(\mathrm{w} / \mathrm{v})$ galactose, and 1\% (w/v) raffinose at an initial optical density of 0.4 at $600 \mathrm{~nm}\left(\mathrm{OD}_{600}\right)$. For coexpression of AtACBP6 and $C z D G A T 1, \mathrm{SC}-\mathrm{Ura}$ in the liquid minimal medium was replaced by synthetic complete medium lacking leucine and uracil. For the fatty acid feeding experiment, yeast cells were cultured in the induction medium with the supplementation of $200 \mathrm{mM}$ of C18:2 or C18:3 fatty acid. Yeast cultures were grown at $30^{\circ} \mathrm{C}$ with shaking at $220 \mathrm{rpm}$.

\section{Preparation of yeast microsomal fractions}

516 Microsomal fractions containing recombinant CzDGAT1 variants were isolated from yeast cells 517 as described previously (Xu et al., 2017; Xu et al., 2019; Xu, Holic, et al., 2018). In brief, the

518 recombinant yeast cells were collected at the mid log growth stage with an OD600 value around 5196.5 , washed, and then resuspended in $1 \mathrm{~mL}$ of lysis buffer [20 mM Tris-HCl pH 7.9, containing $52010 \mathrm{mM} \mathrm{MgCl} 2,1 \mathrm{mM}$ EDTA, 5\% (v/v) glycerol, $300 \mathrm{mM}$ ammonium sulfate and $2 \mathrm{mM}$ 521 dithiothreitol]. The cells were homogenized by a bead beater (Biospec, Bartlesville, OK, USA) 522 in the presence of $0.5 \mathrm{~mm}$ glass beads and then centrifuged at $10000 \mathrm{~g}$ at $4^{\circ} \mathrm{C}$ for $30 \mathrm{~min}$ to 523 remove cell debris and glass beads. The recovered supernatant was further centrifuged at 105 $524000 \mathrm{~g}$ at $4^{\circ} \mathrm{C}$ for $70 \mathrm{~min}$ to pellet the microsomes. The resulting microsomal fractions were 525 resuspended in ice-cold suspension buffer (3 mM imidazole buffer, $\mathrm{pH}$ 7.4, and $125 \mathrm{mM}$ sucrose). Protein concentration was quantified by the Bradford assay (Bio-Rad, Mississauga,

527 Canada) using BSA as a standard (Bradford, 1976).

\section{In vitro DGAT1 assay}

530 In vitro DGAT assay was performed according to the procedure described previously (Xu et al., 531 2017; Xu et al., 2019; Xu, Holic, et al., 2018). Briefly, a 60- $\mu$ L reaction mixture containing 200 $532 \mathrm{mM}$ HEPES-NaOH (pH 7.4), $3.2 \mathrm{mM} \mathrm{MgCl}$, $333 \mu \mathrm{M}$ sn-1,2-diolein [dispersed in 0.2\% (v/v) 533 Tween 20], $15 \mu \mathrm{M}\left[1-{ }^{14} \mathrm{C}\right]$ oleoyl-CoA (56 $\mu \mathrm{Ci} / \mu \mathrm{mol}$; American Radiolabeled Chemicals, St. 534 Louis, MO, USA), and 10-50 $\mu \mathrm{g}$ of microsomal protein was incubated at $30^{\circ} \mathrm{C}$ for $4-30$ min with 535 shaking. The reaction was initiated by adding microsomes containing recombinant CzDGAT1 536 variants and terminated by adding $10 \mu L$ of $10 \%(w / v)$ SDS. The entire reaction mixture was 
then loaded onto a thin-layer chromatography (TLC) plate $(0.25 \mathrm{~mm}$ Silica gel, DC-Fertigplatten,

538 Macherey-Nagel, Germany). The plate was developed with hexane/diethyl ether/acetic acid

539 (80:20:1, v/v/v) and the resolved lipids were visualized by phosphorimaging (Typhoon Trio

540 Variable Mode Imager, GE Healthcare, Mississauga, Canada). The corresponding TAG spots

541 were scraped and quantified for radioactivity using a LS 6500 multi-purpose scintillation counter

542 (Beckman-Coulter, Mississauga, Canada).

For kinetic assay, the concentration of $\left[1-{ }^{14} \mathrm{C}\right]$ oleoyl-CoA was varied from 0.1 to $25 \mu \mathrm{M}$

544 while $s n-1,2$-diolein was held constant at $333 \mu \mathrm{M}$. The DGAT assay reaction time and the

545 quantity of microsomal protein used were as follows: for CzDGAT1 1-550 and ACBP-CzDGAT1 1 -

$546550,10 \mu \mathrm{g}$ of microsomal protein for $4 \mathrm{~min}$; for ACBP-CzDGAT1 ${ }_{81-550}, 5 \mu \mathrm{g}$ of microsomal

547 protein for $30 \mathrm{~min}$; for CzDGAT1 $1_{81-550}, 10 \mu \mathrm{g}$ of microsomal protein for $30 \mathrm{~min}$. Enzyme kinetic

548 parameters were calculated by fitting the data to the Michaelis-Menten equation, allosteric

549 sigmoidal equation, or a previously proposed model accounting for sigmoidicity and substrate

550 inhibition (Xu et al., 2017) using the program GraphPad Prism (version 6.0; GraphPad Software,

551 La Jolla, CA, USA).

\section{Transient expression of $C z D G A T 1$ variants in $N$. benthamiana leaves}

$554 N$. benthamiana plants were grown in a growth chamber at $25^{\circ} \mathrm{C}, 50 \%$ humidity and $16 / 8 \mathrm{hr}$

555 day/night cycle. For transient expression in N. benthamiana leaves for lipid production, cDNAs

556 encoding AtWRI1 (previously isolated using A. thaliana cDNA in our lab), CzDGAT1 ${ }_{1-550}$, the

557 N-terminal truncation mutant (CzDGAT1 1 11-550) and their ACBP-fused versions (ACBP-

558 CzDGAT1 $_{1-550}$ and ACBP-CzDGAT1 ${ }_{81-550)}$ ) were subcloned in a pGREEN 0229 vector under a

559 cauliflower mosaic virus (CaMV) $35 S$ promoter, respectively. For the examination of the

560 subcellular localization, cDNAs encoding CzDGAT1 1-550 and ACBP-CzDGAT1 1-550 were fused

561 in frame to the downstream of Venus, which was amplified from the pSYFP2-SCFP3A plasmid

562 (a gift from Dr. Dorus Gadella; Addgene plasmid \# 22905) (Kremers et al., 2006), and was

563 inserted downstream of a CaMV 35S promoter in the modified pGPTVII vector (kindly provided

564 by Dr. Jörg Kudla, University of Münster) (Becker et al., 1992; Gehl et al., 2009). The coding

565 sequence of AtGPAT9 (Singer et al., 2016) was fused in frame to the upstream of SCFP3A 
566 (amplified from the pSYFP2-SCFP3A plasmid), subcloned in the modified pGPTVII vector 567 under a $C a M V 35 S$ promoter and used as an ER marker (Gidda et al., 2009).

All constructs were individually transformed to Agrobacterium tumefaciens GV3101 cells via electroporation. Each pGREEN construct was transformed along with the pSOUP helper plasmid. A. tumefaciens cultures containing the $p 19$ vector encoding a viral suppressor protein and each variant $C z D G A T 1$ were mixed in a transformation medium [50 mM MES, 2 $\mathrm{mM} \mathrm{Na} \mathrm{PO}_{4}, 0.5 \%(\mathrm{w} / \mathrm{v})$ glucose and $0.1 \mathrm{mM}$ acetosyringone] with the final $\mathrm{OD}_{600}$ of each culture equal to 0.125 (or 0.25 for subcellular localization experiments) prior to infiltration into $N$. benthamiana leaves as described by Vanhercke et al. (2013). For lipid analysis, $N$. benthamiana plants were grown for a further five days before leaf samples were collected, flash frozen, freeze-dried, and stored at $-80^{\circ} \mathrm{C}$. For protein extraction, $N$. benthamiana leaves were harvested after 2 days of infiltration and were ground in liquid nitrogen to a fine powder using mortar and pestle. The resulting leaf powders were then mixed with 1 volume (v/w) of ice-cold extraction buffer (4 M urea, $100 \mathrm{mM}$ DTT, $1 \%$ Triton X-100, and $1 \mathrm{mM} \mathrm{PMSF}$ ), and incubated on ice for $10 \mathrm{~min}$. The homogenate was clarified by centrifugation at $12000 \mathrm{~g}$ for $5 \mathrm{~min}$ at $4{ }^{\circ} \mathrm{C}$ and the supernatant was used for SDS-PAGE gel analysis immediately after preparation.

For subcellular localization experiments, AtGPAT9-SCFP3C was co-infiltrated with the p19 vector and Venus-CzDGAT1 or Venus-ACBP-CzDGAT1 and the fluorescence of the lower epidermis of leaves after 2-3 days of infiltration was visualized using a fluorescent microscope (Axio Imager M1m microscope; Carl Zeiss Inc., Germany). The excitation wavelengths for

586 Venus and SCFP3A were 546/12 and $365 \mathrm{~nm}$, respectively, and the emission filter wavelengths were 575-640 nm for Venus and 455/50 nm for SCFP3A. For the quantification of fluorescence intensity, the fluorescent proteins were extracted from tobacco leaves transiently expressing the p19 vector and Venus-CzDGAT1 or Venus-ACBP-CzDGAT1 by grinding on ice with a two-fold volume of pre-chilled extraction buffer containing $100 \mathrm{mM}$ MOPS ( $\mathrm{pH} 7.2), 5 \mathrm{mM} \mathrm{MgCl}$,

$591 \quad 0.02 \%$ BSA and $1 \%$ protease inhibitor, followed by centrifugation. One hundred microliters of 592 the supernatant were then placed into 96-well solid black plates (Corning Inc., Corning, NY, 593 USA) and the fluorescence intensity of Venus-tagged protein was measured on a Synergy H4 594 Hybrid reader (Biotek, Winooskit, VT, USA) at excitation and emission wavelengths of $485 \mathrm{~nm}$ 595 and $528 \mathrm{~nm}$, respectively. 


\section{Western blotting}

597 Equivalent amounts of yeast microsomal proteins $(15 \mu \mathrm{g})$ or protein extracts from leaf samples containing recombinant CzDGAT1 variants were incubated with 5× SDS loading buffer at room temperature for $15 \mathrm{~min}$, resolved through SDS-PAGE Gels (Bio-Rad) and electrotransfered (2 h at $80 \mathrm{~V}$ or $16 \mathrm{~h}$ at $30 \mathrm{~V}$ and $4^{\circ} \mathrm{C}$ ) onto polyvinylidene difluoride membrane (Amersham, GE Healthcare). The recombinant enzymes were probed using anti-V5-HRP conjugated antibody

602 (Invitrogen), which was detected using an ECL Advance Western Blotting Detection Kit 603 (Amersham) by a FluorChem SP imager (Alpha Innotech Corp., San Leandro, CA, U.S.A.). The band densities were semi-quantified with ImageJ software (Schneider et al., 2012).

\section{Lipid analysis}

607 The yeast neutral lipid content was analyzed by the Nile red fluorescence assay as described 608 previously (Xu et al., 2017). In brief, an aliquot (100 $\mu \mathrm{L}$ ) of yeast culture was incubated with 5 $609 \mu \mathrm{L}$ of Nile red solution $(0.1 \mathrm{mg} / \mathrm{mL}$ in methanol) into 96 -well solid black plates (Corning Inc.).

610 The fluorescence was measured before and after the addition of the Nile red solution with 611 excitation and emission at 485 and $538 \mathrm{~nm}$, respectively, using a Synergy H4 Hybrid reader

612 (Biotek). The neutral lipid content in yeast was represented by the Nile red values which were 613 calculated based on the change in fluorescence over $\mathrm{OD}_{600}\left(\Delta \mathrm{F} / \mathrm{OD}_{600}\right)$.

615 samples were analyzed using GC/MS. Yeast total lipids were extracted from approximately 30

$616 \mathrm{mg}$ of lyophilized yeast cells as described previously (Xu et al., 2019; Xu, Holic, et al., 2018).

617 As for $N$. benthamiana leaf samples, $70 \mathrm{mg}$ lyophilized leaf tissues were homogenized in

618 chloroform: isopropanol (2:1, v/v) for total lipids extraction as described previously

619 (Mietkiewska et al., 2014). For quantification, $100 \mu \mathrm{g}$ of triheptadecanoin (C17:0 TAG) were 620 added to each sample as an internal standard. The extracted lipids were further separated on a 621 TLC plate (0.25 mm Silica gel, DC-Fertigplatten) as described above and the lipid bands were 622 visualized by spraying with primulin solution [0.05\% primulin (w/v) in acetone/water (80:20, $623 \mathrm{v} / \mathrm{v})]$. The corresponding TAG bands were then scraped and trans-methylated by incubating with $6241 \mathrm{~mL}$ of $3 \mathrm{~N}$ methanolic $\mathrm{HCl}$ at $80{ }^{\circ} \mathrm{C}$ for $1 \mathrm{~h}$. The resulting fatty acid methyl esters were 625 analyzed using GC/MS (Agilent Technologies, Wilmington, DE) equipped with a capillary DB- 
23 column $(30 \mathrm{~m} \times 0.25 \mathrm{~mm} \times 0.25 \mu \mathrm{m})$ as described previously $(\mathrm{Xu}$ et al., 2019; Xu, Holic, et

$627 a l ., 2018)$.

\section{Statistical analysis}

630 Data are shown as means \pm standard derivation (S.D.) for the number of independent

631 experiments indicated. Significant differences between two groups were assessed using a

632 Student's t-test with the SPSS statistical package (SPSS 16.0, Chicago, IL, USA). The equality

633 of variance was tested by Levene's test. The unpaired Student's t-test assuming equal variances

634 and the unpaired Student's t-test with Welch corrections assuming unequal variances were

635 performed when the variances were equal and unequal, respectively.

\section{ACCESSION NUMBERS}

638 Sequence data from this article can be accessed in the Phytozome/GenBank/Arabidopsis

639 Genome Initiative databases under the following accession numbers: AtACBP6, NM_102916;

640 AtGPAT9, AT5G60620; AtWRI1, AY254038; CzDGAT1, Cz09g08290.

\section{ACKNOWLEDGEMENTS}

643 We acknowledge the support provided by the University of Alberta Start-up Research Grant,

644 Natural Sciences and Engineering Research Council of Canada Discovery Grant (RGPIN-2016-

645 05926) and the Canada Research Chairs Program. We are grateful to Dr. Mee-Len Chye

646 (University of Hong Kong) for providing the plasmid pAT332 containing the coding sequence of

647 AtACBP6 and Dr. Igor Stagljar (University of Toronto) for providing the membrane yeast two-

648 hybrid system. We also thank Dr. Stacy Singer (Agriculture and Agri-Food Canada) for sharing

649 her experience on tobacco leaf infiltration, Dr. Michael Gänzle, Dr. Yuan Fang and Mr. Kosala

650 Waduthanthri (University of Alberta) for their assistance in fluorescence microscopy and Dr.

651 Shanjida Khan (University of Alberta) for providing the N. benthamiana seeds. 


\section{AUTHOR CONTRIBUTIONS}

655 GC supervised the experiment; YX, GC, and KMPC designed the experiment; YX performed the 656 experiments and prepared the initial draft of the manuscript. YX, KMPC and LF analyzed the 657 data. KMPC and KJ contributed valuable discussion during this study. All authors were involved 658 in further editing of the manuscript.

\section{CONFLICT OF INTEREST}

662 The authors declare that they have no conflicts of interest with the content of this article.

\section{SUPPORTING INFORMATION}

665 Method S1. Membrane yeast two-hybrid assay.

666 Table S1. DGAT1 proteins used for multiple sequence alignment.

667 Table S2. Apparent kinetic parameters of CzDGAT1 variants using a combined model

668 accounting for sigmoidicity and substrate inhibition (Xu et al., 2017).

669 Table S3. Primers used in the current study.

670 Figure S1. Alignment of DGAT1 from different species.

671 Figure S2. Prediction of intrinsic disorder profile (blue) of the N-terminal region of DGAT1

672 from representative algae, plant and animals and its likelihood to participate in protein-protein 673 interaction (red).

674 Figure S3. DGAT activity of CzDGAT1 variant enzymes at high oleoyl-CoA concentrations.

675 Figure S4. Probing possible self-interaction of CzDGAT1 variants using membrane yeast two676 hybrid assay.

677 Figure S5. Enzyme activity of CzDGAT1 variants in the presence of Coenzyme A (CoA).

678 Figure S6. Illustration of the N-terminal fusion of acyl-CoA binding protein (ACBP) to 679 CzDGAT1. 
REFERENCES:

682

683

684

Alameldin, H., Izadi-Darbandi, A., Smith, S.A. and Balan, V. (2017) Metabolic engineering to increase the corn seed storage lipid quantity and change its compositional quality. Crop Sci, 57, 1854-1864.

Becker, D., Kemper, E., Schell, J. and Masterson, R. (1992) New plant binary vectors with selectable markers located proximal to the left T-DNA border. Plant Mol. Biol., 20, 11951197.

Bouvier-Navé, P., Benveniste, P., Oelkers, P., Sturley, S.L. and Schaller, H. (2000) Expression in yeast and tobacco of plant cDNAs encoding acyl CoA:diacylglycerol acyltransferase. FEBS J., 267, 85-96.

Bradford, M.M. (1976) A rapid and sensitive method for the quantitation of microgram quantities of protein utilizing the principle of protein-dye binding. Anal. Biochem., 72, 248254.

Brown, A.P., Johnson, P., Rawsthorne, S. and Hills, M.J. (1998) Expression and properties of acyl-CoA binding protein from Brassica napus. Plant Physiol. Biochem., 36, 629-635.

Caldo, K.M.P., Acedo, J.Z., Panigrahi, R., et al. (2017) Diacylglycerol acyltransferase 1 is regulated by its $\mathrm{N}$-terminal domain in response to allosteric effectors. Plant Physiol, 175, $667-680$.

Capella-Gutiérrez, S., Silla-Martínez, J.M. and Gabaldón, T. (2009) trimAl: A tool for automated alignment trimming in large-scale phylogenetic analyses. Bioinformatics, 25, 1972-1973.

Chen, G., Xu, Y., Siloto, R.M.P., et al. (2017) High-performance variants of plant diacylglycerol acyltransferase 1 generated by directed evolution provide insights into structure function. Plant J, 92, 167-177.

Chen, J.E. and Smith, A.G. (2012) A look at diacylglycerol acyltransferases (DGATs) in algae. J. Biotechnol., 162, 28-39.

Chen, Q.-F., Xiao, S. and Chye, M.-L. (2008) Overexpression of the Arabidopsis 10-kilodalton acyl-Coenzyme A-binding protein ACBP6 enhances freezing tolerance. Plant Physiol., 148, 
304-315.

710

711

712

713

Du, Z.Y., Arias, T., Meng, W. and Chye, M.L. (2016) Plant acyl-CoA-binding proteins: An emerging family involved in plant development and stress responses. Prog. Lipid Res., 63, 165-181.

Ecker, D.J., Stadel, J.M., Butt, T.R., et al. (1989) Increasing gene expression in yeast by fusion to ubiquitin. J. Biol. Chem., 264, 7715-7719.

Elleuche, S. (2015) Bringing functions together with fusion enzymes - from nature's inventions to biotechnological applications. Appl. Microbiol. Biotechnol., 99, 1545-1556.

Gehl, C., Waadt, R., Kudla, J., Mendel, R.R. and Hänsch, R. (2009) New GATEWAY vectors for high throughput analyses of protein-protein interactions by bimolecular fluorescence complementation. Mol. Plant, 2, 1051-1058.

Gidda, S.K., Shockey, J.M., Rothstein, S.J., Dyer, J.M. and Mullen, R.T. (2009) Arabidopsis thaliana GPAT8 and GPAT9 are localized to the ER and possess distinct ER retrieval signals: Functional divergence of the dilysine ER retrieval motif in plant cells. Plant Physiol. Biochem., 47, 867-879.

Gong, Y., Zhang, J., Guo, X., Wan, X., Liang, Z., Hu, C.J. and Jiang, M. (2013) Identification and characterization of PtDGAT2B, an acyltransferase of the DGAT2 acylCoenzyme A: Diacylglycerol acyltransferase family in the diatom Phaeodactylum tricornutum. FEBS Lett., 587, 481-487.

\section{Greer, M.S., Truksa, M., Deng, W., Lung, S.C., Chen, G. and Weselake, R.J. (2015)} Engineering increased triacylglycerol accumulation in Saccharomyces cerevisiae using a modified type 1 plant diacylglycerol acyltransferase. Appl. Microbiol. Biotechnol., 99, $2243-2253$.

Guihéneuf, F., Leu, S., Zarka, A., et al. (2011) Cloning and molecular characterization of a novel acyl-CoA:diacylglycerol acyltransferase 1-like gene (PtDGAT1) from the diatom Phaeodactylum tricornutum. FEBS J., 278, 3651-3666.

Guindon, S., Dufayard, J.-F.F., Lefort, V., Anisimova, M., Hordijk, W. and Gascuel, O. (2010) New algorithms and methods to estimate maximum-likelihood phylogenies: Asessing the performance of PhyML 2.0. Syst. Biol., 59, 307-321. 
Guo, X., Fan, C., Chen, Y., Wang, J., Yin, W., Wang, R.R.C. and Hu, Z. (2017) Identification and characterization of an efficient acyl-CoA: diacylglycerol acyltransferase 1 (DGAT1) gene from the microalga Chlorella ellipsoidea. BMC Plant Biol., 17, 48.

Hondred, D., Walker, J.M., Mathews, D.E. and Vierstra, R.D. (1999) Use of ubiquitin fusions to augment protein expression in transgenic plants. Plant Physiol., 119, 713-723.

Hu, Q., Sommerfeld, M., Jarvis, E., Ghirardi, M., Posewitz, M., Seibert, M. and Darzins, A.

Iwai, M., Ikeda, K., Shimojima, M. and Ohta, H. (2014) Enhancement of extraplastidic oil synthesis in Chlamydomonas reinhardtii using a type-2 diacylglycerol acyltransferase with a phosphorus starvation-inducible promoter. Plant Biotechnol. J., 12, 808-819.

Jacquet, A., Daminet, V., Haumont, M., Garcia, L., Chaudoir, S., Bollen, A. and Biemans, (2008) Microalgal triacylglycerols as feedstocks for biofuel production: perspectives and advances. Plant J., 54, 621-639.

Jako, C., Kumar, A., Wei, Y., Zou, J., Barton, D.L., al., et, Giblin, E.M., Covello, P.S. and

Katavic, V., Reed, D.W., Taylor, D.C., Ciblin, E.M., Barton, D.L., Zou, J., Mackenzie, S.L.,

R. (1999) Expression of a recombinant Toxoplasma gondii ROP2 fragment as a fusion protein in bacteria circumvents insolubility and proteolytic degradation. Protein Expr. Purif., 17, 392-400. Taylor, D.C. (2001) Seed-specific over-expression of an Arabidopsis cDNA encoding a diacylglycerol acyltransferase enhances seed oil content and seed weight. Plant Physiol., 126, 861-874.

Katoh, K. and Standley, D.M. (2013) MAFFT multiple sequence alignment software version 7: Improvements in performance and usability. Mol. Biol. Evol., 30, 772-780.

Kim, H., Park, J.H., Kim, A.Y., Suh, M.C., Kim, D.J., Kim, Y.A. and Suh, M.C. (2016) methanesulfonate-induced mutation in Arabidopsis thaliana affecting diacylglycerol acyltransferase activity. Plant Physiol., 108, 399-409.

Functional analysis of diacylglycerol acyltransferasel genes from Camelina sativa and effects of $C S D G A T 1 B$ overexpression on seed mass and storage oil content in $C$. sativa. Plant Biotechnol. Rep., 10, 141-153. 
Kirchner, L., Wirshing, A., Kurt, L., Reinard, T., Glick, J., Cram, E.J., Jacobsen, H.-J. and Lee-Parsons, C.W.T. (2016) Identification, characterization, and expression of diacylgylcerol acyltransferase type-1 from Chlorella vulgaris. Algal Res., 13, 167-181.

Knudsen, J., Faergeman, N.J., Skøtt, H., et al. (1994) Yeast acyl-CoA-binding protein: acylCoA-binding affinity and effect on intracellular acyl-CoA pool size. Biochem. J., 302, 479485.

Kong, F., Romero, I.T., Warakanont, J. and Li-Beisson, Y. (2018) Lipid catabolism in microalgae. New Phytol., 218, 1340-1348.

Kremers, G., Goedhart, J., Munster, E.B. Van and Gadella, T.W.J. (2006) Cyan and yellow super fluorescent proteins with improved brightness, protein folding, and FRET Förster radius. Biochemistry, 45, 6570-6580.

Krink-Koutsoubelis, N., Loechner, A.C., Lechner, A., et al. (2018) Engineered poduction of short-chain acyl-Coenzyme A esters in Saccharomyces cerevisiae. ACS Synth. Biol., 7, 1105-1115.

Krogh, A., Larsson, B., Heijne, G. von and Sonnhammer, E. (2001) Predicting transmembrane protein topology with a hidden Markov model: Application to complete genomes. J. Mol. Biol., 305, 567-580.

Kroon, J.T.M., Wei, W., Simon, W.J. and Slabas, A.R. (2006) Identification and functional expression of a type 2 acyl-CoA:diacylglycerol acyltransferase (DGAT2) in developing

Lardizabal, K., Effertz, R., Levering, C., Mai, J., Pedroso, M.C., Jury, T., Aasen, E., Gruys, castor bean seeds which has high homology to the major triglyceride biosynthetic enzyme

Larson, T.R. and Graham, I. a. (2001) A novel technique for the sensitive quantification of acyl CoA esters from plant tissues. Plant J., 25, 115-125.

Letunic, I. and Bork, P. (2016) Interactive tree of life (iTOL) v3: An online tool for the display and annotation of phylogenetic and other trees. Nucleic Acids Res., 44, W242-W245. 
Li, R., Yu, K. and Hildebrand, D.F. (2010) DGAT1, DGAT2 and PDAT expression in seeds and other tissues of epoxy and hydroxy fatty acid accumulating plants. Lipids, 45, 145-57.

Li, R., Yu, K., Wu, Y., et al. (2012) Vernonia DGATs can complement the disrupted oil and protein metabolism in epoxygenase-expressing soybean seeds. Metab. Eng., 14, 29-38.

Liu, B. and Benning, C. (2013) Lipid metabolism in microalgae distinguishes itself. Curr Opin Biotechnol, 24, 300-309.

Liu, J., Han, D., Yoon, K., Hu, Q. and Li, Y. (2016) Characterization of type 2 diacylglycerol acyltransferases in Chlamydomonas reinhardtii reveals their distinct substrate specificities and functions in triacylglycerol biosynthesis. Plant J., 86, 3-19.

Liu, Q., Siloto, R.M.P., Lehner, R., Stone, S.J. and Weselake, R.J. (2012) Acyl-

Mandrup, S., Jepsen, R., Skøtt, H., Rosendal, J., Højrup, P., Kristiansen, K. and Knudsen,

Liu, J., Sun, Z., Mao, X., Gerken, H., Wang, X. and Yang, W. (2019) Multi-omics analysis reveals distinct mechanism of oleaginousness in the emerging model alga Chromochloris zofingiensis. Plant J., 98, 1060-1077.

Mao, X., Wu, T., Kou, Y., Shi, Y., Zhang, Y. and Liu, J. (2019) Characterization of type I and J. (1993) Effect of heterologous expression of acyl-CoA-binding protein on acyl-CoA level

Marsh, J.A., Stadel, J.M., Ecker, D.J. and Crooke, S.T. (1989) Ubiquitin fusion augments the yield of cloned gene products in Escherichia coli. Proc. Natl. Acad. Sci., 86, 2540-2544.

McFie, P.J., Stone, S.L., Banman, S.L. and Stone, S.J. (2010) Topological orientation of acyltype II diacylglycerol acyltransferases from the emerging model alga Chlorella zofingiensis reveals their functional complementarity and engineering potential. Biotechnol. Biofuels, 12, 28. CoA:diacylglycerol acyltransferase-1 (DGAT1) and identification of a putative active site histidine and the role of the $\mathrm{n}$ terminus in dimer/tetramer formation. J. Biol. Chem., 285, $37377-37387$. 
Miao, R., Lung, S.-C., Li, X., Li, X.D. and Chye, M.-L. (2019) Thermodynamic insights into an interaction between ACYL-COA-BINDING PROTEIN2 and LYSOPHOSPHOLIPASE2 in Arabidopsis. J. Biol. Chem., 294, 6214-6226.

Mietkiewska, E., Miles, R., Wickramarathna, A., Sahibollah, A.F., Greer, M.S., Chen, G. and Weselake, R.J. (2014) Combined transgenic expression of Punica granatum conjugase $(F A D X)$ and FAD2 desaturase in high linoleic acid Arabidopsis thaliana mutant leads to increased accumulation of punicic acid. Planta, 240, 575-583.

Mishra, S., Yadav, D.K. and Tuli, R. (2006) Ubiquitin fusion enhances cholera toxin B subunit expression in transgenic plants and the plant-expressed protein binds GM1 receptors more efficiently. J. Biotechnol., 127, 95-108.

Moreno, A.J., Venegas, M., Vaistij, F.E., Salas, J.J., Larson, T.R., Garcés, R., Graham, I.A. and Martínez, E. (2014) Effect of a mutagenized acyl-ACP thioesterase FATA allele from sunflower with improved activity in tobacco leaves and Arabidopsis seeds. Planta, 239, 667-677.

O'Quin, J.B., Mullen, R.T. and Dyer, J.M. (2009) Addition of an N-terminal epitope tag significantly increases the activity of plant fatty acid desaturases expressed in yeast cells. Appl. Microbiol. Biotechnol., 83, 117-125.

Oakes, J., Brackenridge, D., Colletti, R., et al. (2011) Expression of fungal diacylglycerol acyltransferase 2 genes to increase kernel oil in maize. Plant Physiol., 155, 1146-1157.

Pryor, K.D. and Leiting, B. (1997) High-level expression of soluble protein in Escherichia coli using a His 6 -tag and maltose-binding-protein double-affinity fusion system. Protein Expr. Purif., 10, 309-319.

Rahman, H., Harwood, J. and Weselake, R. (2013) Increasing seed oil content in Brassica species through breeding and biotechnology. Lipid Technol., 25, 182-185.

Ro, D., Paradise, E.M., Ouellet, M., et al. (2006) Production of the antimalarial drug precursor artemisinic acid in engineered yeast. Nature, 440, 940-943.

Robinson, C. V, Chung, E.W., Kragelund, B.B., Knudsen, J., Aplin, R.T., Poulsen, F.M. and Dobson, C.M. (1996) Probing the nature of noncovalent interactions by mass spectrometry. A study of protein - CoA ligand binding and assembly. J. Am. Chem. Soc., 
118, 8646-8653.

853

Roesler, K., Shen, B., Bermudez, E., et al. (2016) An improved variant of soybean type 1 diacylglycerol acyltransferase increases the oil content and decreases the soluble carbohydrate content of soybeans. Plant Physiol., 171, 878-893.

Roth, M.S., Cokus, S.J., Gallaher, S.D., et al. (2017) Chromosome-level genome assembly and transcriptome of the green alga Chromochloris zofingiensis illuminates astaxanthin production. Proc. Natl. Acad. Sci. U. S. A., 114, E4296-E4305.

Sandager, L., Gustavsson, M.H., Stahl, U., Dahlqvist, A., Wiberg, E., Banas, A., Lenman, M., Ronne, H. and Stymne, S. (2002) Storage lipid synthesis is non-essential in yeast. $J$ Biol Chem, 277, 6478-6482.

Schjerling, C.K., Hummel, R., Hansen, J.K., Børsting, C., Mikkelsen, J.M., Kristiansen, R. and Knudsen, J. (1996) Disruption of the gene encoding the acyl-CoA-binding protein (ACB1) perturbs acyl-CoA metabolism in Saccharomyces cerevisiae. J. Biol. Chem., 271, 22514-22521.

Schneider, C.A., Rasband, W.S. and Eliceiri, K.W. (2012) NIH Image to ImageJ: 25 years of image analysis. Nat. Methods, 9, 671-675.

Shockey, J.M., Gidda, S.K., Chapital, D.C., Kuan, J.-C.C., Dhanoa, P.K., Bland, J.M., Rothstein, S.J., Mullen, R.T. and Dyer, J.M. (2006) Tung tree DGAT1 and DGAT2 have nonredundant functions in triacylglycerol biosynthesis and are localized to different subdomains of the endoplasmic reticulum. Plant Cell, 18, 2294-2313.

Siloto, R.M.P., Madhavji, M., Wiehler, W.B., Burton, T.L., Boora, P.S., Laroche, A. and Weselake, R.J. (2008) An N-terminal fragment of mouse DGAT1 binds different acylCoAs with varying affinity. Biochem. Biophys. Res. Commun., 373, 350-354.

Singer, S.D., Chen, G., Mietkiewska, E., Tomasi, P., Jayawardhane, K., Dyer, J.M. and Weselake, R.J. (2016) Arabidopsis GPAT9 contributes to synthesis of intracellular glycerolipids but not surface lipids. J. Exp. Bot., 67, 4627-4638.

Sriram, S.M., Kim, B.Y. and Kwon, Y.T. (2011) The N-end rule pathway: Emerging functions and molecular principles of substrate recognition. Nat. Rev. Mol. Cell Biol., 12, 735-747. 
Streatfield, S.J. (2007) Approaches to achieve high-level heterologous protein production in plants. Plant Biotechnol. J., 5, 2-15.

Tian, L. and Sun, S.S.M. (2011) Ubiquitin fusion expression and tissue-dependent targeting of hG-CSF in transgenic tobacco. BMC Biotechnol., 11, 91.

Turchetto-Zolet, A.C., Maraschin, F.S., Morais, G.L. De, Cagliari, A., Andrade, C.M., Margis-Pinheiro, M. and Margis, R. (2011) Evolutionary view of acyl-CoA diacylglycerol acyltransferase (DGAT), a key enzyme in neutral lipid biosynthesis. BMC Evol. Biol., 11, 263.

Tzen, J., Cao, Y., Laurent, P., Ratnayake, C. and Huang, A. (1993) Lipids, proteins, and

Vanhercke, T., Divi, U.K., Tahchy, A. El, et al. (2017) Step changes in leaf oil accumulation structure of seed oil bodies from diverse species. Plant Physiol., 101, 267-276.

Vanhercke, T., Tahchy, A. El, Shrestha, P., Zhou, X.-R.R., Singh, S.P. and Petrie, J.R.

Ward, J.J., McGuffin, L.J., Bryson, K., Buxton, B.F. and Jones, D.T. (2004) The DISOPRED server for the prediction of protein disorder. Bioinformatics, 20, 2138-2139.

Wei, H., Shi, Y., Ma, X., Pan, Y., Hu, H., Li, Y., Luo, M., Gerken, H. and Liu, J. (2017) A type-I diacylglycerol acyltransferase modulates triacylglycerol biosynthesis and fatty acid composition in the oleaginous microalga, Nannochloropsis oceanica. Biotechnol. Biofuels, $10,174$. (1993) Developmental profile of diacylglycerol acyltransferase in maturing seeds of oilseed rape and safflower and microspore-derived cultures of oilseed rape. Plant Physiol., 102, 565-571. 
Weselake, R.J., Shah, S., Tang, M., et al. (2008) Metabolic control analysis is helpful for informed genetic manipulation of oilseed rape (Brassica napus) to increase seed oil content. J. Exp. Bot., 59, 3543-3549.

Xin, Y., Lu, Y., Lee, Y.-Y.Y., et al. (2017) Producing designer oils in industrial microalgae by rational modulation of co-evolving type-2 diacylglycerol acyltransferases. Mol. Plant, 10, $1523-1539$.

Xin, Y., Shen, C., She, Y., et al. (2018) Biosynthesis of triacylglycerol molecules with tailored PUFA profile in industrial microalgae. Mol. Plant.

Xu, Y., Caldo, K.M.P., Pal-Nath, D., Ozga, J., Lemieux, M.J., Weselake, R.J. and Chen, G. (2018) Properties and biotechnological applications of acyl-CoA:diacylglycerol acyltransferase and phospholipid:diacylglycerol acyltransferase from terrestrial plants and microalgae. Lipids, 53, 663-688.

Xu, Y., Chen, G., Greer, M.S., et al. (2017) Multiple mechanisms contribute to increased neutral lipid accumulation in yeast producing recombinant variants of plant diacylglycerol acyltransferase 1. J. Biol. Chem., 292, 17819-17831.

Xu, Y., Falarz, L. and Chen, G. (2019) Characterization of type-2 diacylglycerol acyltransferases in the green microalga Chromochloris zofingiensis. J. Agric. Food Chem., 67, 291-298.

Xu, Y., Holic, R., Li, D., Pan, X., Mietkiewska, E., Chen, G., Ozga, J., Weselake, R.J. and Holic, R. (2018) Substrate preferences of long-chain acyl-CoA synthetase and diacylglycerol acyltransferase contribute to enrichment of flax seed oil with $\alpha$-linolenic acid. Biochem. J., 475, 1473-1489.

Yurchenko, O.P., Nykiforuk, C.L., Moloney, M.M., Ståhl, U., Banaś, A., Stymne, S. and Weselake, R.J. (2009) A 10-kDa acyl-CoA-binding protein (ACBP) from Brassica napus enhances acyl exchange between acyl-CoA and phosphatidylcholine. Plant Biotechnol. J., 7, $602-610$.

Yurchenko, O.P. and Weselake, R.J. (2011) Involvement of low molecular mass soluble acylCoA-binding protein in seed oil biosynthesis. N. Biotechnol., 28, 97-109.

Zou, J., Wei, Y., Jako, C., Kumar, A., Selvaraj, G. and Taylor, D.C. (1999) The Arabidopsis 
937 thaliana TAG1 mutant has a mutation in a diacylglycerol acyltransferase gene. Plant J., 19, 938 645-653.

939 Zulu, N.N., Popko, J., Zienkiewicz, K., Tarazona, P., Herrfurth, C. and Feussner, I. (2017)

$940 \quad$ Heterologous co-expression of a yeast diacylglycerol acyltransferase (ScDGA1) and a plant 941 oleosin (AtOLEO3) as an efficient tool for enhancing triacylglycerol accumulation in the

942 marine diatom Phaeodactylum tricornutum. Biotechnol. Biofuels, 10, 187.

943

944 


\section{TABLES}

946

947 Table 1. Apparent kinetic parameters of CzDGAT1 variants. DGAT activity was examined

948 at increasing oleoyl-CoA concentration from 0.1 to 7.5 or $10 \mu \mathrm{M}$. Data were fitted to a nonlinear

949 regression using allosteric sigmoidal equation with GraphPad Prism software. Data shown are

950 means \pm S.D. $(n=3)$.

951

\begin{tabular}{|c|c|c|c|c|}
\hline Enzyme & DGAT1 $1_{1-550}$ & DGAT1 $_{81-550}$ & $\begin{array}{c}\text { ACBP- } \\
\text { DGAT1 }_{1-550}\end{array}$ & $\begin{array}{c}\text { ACBP- } \\
\text { DGAT1 } 181-550\end{array}$ \\
\hline \multicolumn{5}{|l|}{ Apparent $\mathrm{V}_{\max }$ (pmol } \\
\hline $\begin{array}{c}\mathrm{TAG} / \mathrm{min} / \mathrm{mg} \\
\text { protein) }\end{array}$ & $276.8 \pm 6.94$ & $17.14 \pm 0.28$ & $436.7 \pm 10.1$ & $34.54 \pm 1.27$ \\
\hline Hill coefficient & $1.39 \pm 0.06$ & $1.71 \pm 0.06$ & $1.91 \pm 0.12$ & $1.57 \pm 0.14$ \\
\hline Apparent $\mathrm{S}_{0.5}(\mu \mathrm{M})$ & $1.48 \pm 0.07$ & $1.83 \pm 0.05$ & $0.94 \pm 0.04$ & $1.50 \pm 0.10$ \\
\hline Goodness of Fit/R ${ }^{2}$ & 0.994 & 0.995 & 0.981 & 0.973 \\
\hline
\end{tabular}

952

953 
FIGURE LEGENDS

955 Figure 1. Phylogenetic relationship among CzDGAT1 and DGAT1 from other organisms.

956 The organism and Phytozome/GenBank accession number/JGI protein ID for each protein

957 sequence are shown as follows: Auxenochlorella protothecoides, Ap, ApDGAT1

958 (XP_011402032); Arabidopsis thaliana, At, AtDGAT1 (NM_127503); Brassica napus, Bn,

959 BnDGAT1 (JN224473); Bos taurus, Bt, BtDGAT1 (AAL49962); Caenorhabditis elegans, Cae,

960 CaeDGAT1 (NM_001269372); Chlorella ellipsoidea, Che, CheDGAT1 (KT779429);

961 Chlamydomonas reinhardtii, Cre, CreDGAT1 (Cre01.g045903); Camelina sativa, Cs,

962 CsDGAT1 (XM_010417066); Coccomyxa subellipsoidea C-169, Csu, CsuDGAT1 (54084);

963 Chlorella vulgaris, Cv, CvDGAT1 (ALP13863); Chromochloris zofingiensis, Cz, CzDGAT1A

964 (MH523419), CzDGAT1B (Cz09g08290); Drosophila melanogaster, Dm, DmDGAT1

965 (AF468649); Danio rerio, Dr, DrDGAT1 (NM_199730); Euonymus alatus, Ea, EaDGAT1

966 (AY751297); Glycine max, Gm, GmDGAT1 (AY496439); Helianthus annuus, Ha, HaDGAT1

967 (HM015632); Homo sapiens, Hs, HsDGAT1 (NM_012079); Jatropha curcas, Jc, JcDGAT1

968 (DQ278448); Klebsormidium nitens, Kn, KnDGAT1 (GAQ91878); Linum usitatissimum, Lu,

969 LuDGAT1 (KC485337); Monodelphis domestica, Md, MdDGAT1 (XM_007488766); Mus

970 musculus, Mm, MmDGAT1 (AF078752); Medicago truncatula, Mt, MtDGAT1

971 (XM_003595183); Nannochloropsis oceanica, No, NoDGAT1 (KY073295); Nicotiana tabacum,

$972 N t$, NtDGAT1 (AF129003); Nematostella vectensis, Nv, NvDGAT1 (XM_001639301); Olea

973 europae, Oe, OeDGAT1 (AY445635); Oryza sativa, Os, OsDGAT1 (NM_001061404);

974 Paracoccidioides brasiliensis, Pb, PbDGAT1 (EEH17170); Perilla frutescens, Pf, PfDGAT1

975 (AF298815); Populus trichocarpa, Pot, PotDGAT1 (XM_006371934); Physcomitrella patens,

976 Pp, PpDGAT1 (XM_001770877); Phaeodactylum tricornutum, Pt, PtDGAT1 (HQ589265);

977 Ricinus communis, Rc, RcDGAT1 (NM_001323734); Rattus norvegicus, Rn, RnDGAT1

978 (AB062759); Sesamum indicum, Si, SiDGAT1 (JF499689); Sus scrofa, Ss, SsDGAT1

979 (NM_214051); Trichoplax adhaerens, Ta, TaDGAT1 (XM_002111989); Toxoplasma gondii, Tg,

980 TgDGAT1 (AY327327); Tropaeolum majus, Tm, TmDGAT1 (AY084052); Thalassiosira

981 pseudonana, Tp, TpDGAT1 (XM_002287179); Vernicia fordii, Vf, VfDGAT1 (DQ356680);

982 Vernonia galamensis, Vg, VgDGAT1 (EF653276); Vitis vinifera, Vv, VvDGAT1 (CAN80418);

983 Yarrowia lipolytica, Yl, YlDGAT1 (XM_502557); and Zea mays, Zm, ZmDGAT1 (EU039830).

984 DGAT1s from the animal, fungal, green algal, and plant groups are shown in orange, blue, pink 
and green, respectively. Algal DGAT1s are shown by red bars. The length of the N-terminus of

986 DGAT1 is shown as a heat map (circle).

Figure 2. CzDGAT1 encodes an active enzyme and has a unique $\mathbf{N}$-terminus with less propensity to become disordered. A and B, Triacylglycerol (TAG) content (A) and fatty acid (FA) composition (B) in yeast producing CzDGAT1 cultured in the absence of FA or the

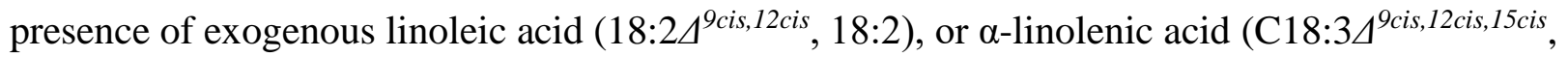

992 18:3). Yeast cells were harvested after $48 \mathrm{~h}$ of induction for lipid analysis. C16:0, Palmitic acid;

993 C18:0, Stearic acid; C18:1, Oleic acid. C, In vitro DGAT assay using yeast microsomal fractions

994 containing recombinant CzDGAT1. D, Sequence alignment of the N-terminal regions of DGAT1.

995 At, Arabidopsis thaliana; Bn, Brassica napus; $C v$, Chlorella vulgaris; $C z$, Chromochloris

996 zofingiensis; No, Nannochloropsis oceanica. E and F, Prediction of intrinsic disorder profile

997 (blue) of the N-terminal regions of CzDGAT1 (D) and BnDGAT1 (E) and likelihood to

998 participate in protein-protein interaction (red) by DISOPRED analysis (Ward et al., 2004). For A,

999 B and C, data represent means \pm S.D. $(n=3)$.

1000

1001 Figure 3. Truncation analysis of the N-terminal domain CzDGAT1. A, Predicted topology of 1002 CzDGAT1 by TMHMM (Krogh et al., 2001). CzDGAT1 has a 107-amino acid residue-long 1003 hydrophilic N-terminal region, followed by 9 predicted transmembrane domains (TMDs). The

1004 numbers indicate the different truncation points. B, Protein production level of the full-length 1005 (CzDGAT1 1-550) and N-terminal truncated (CzDGAT1 ${ }_{81-550}$ and CzDGAT1 107-550) CzDGAT1 1006 variant proteins. The enzyme amount was semi-quantified by Image J software (Schneider et al.,

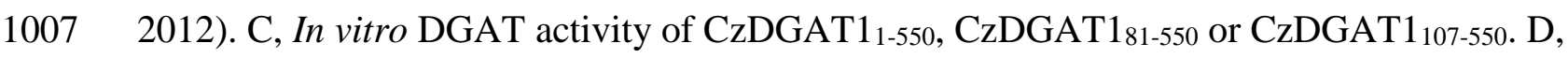
1008 Normalized DGAT activity of CzDGAT1 1-550, CzDGAT1 $181-550$ or CzDGAT1 107-550. Normalized 1009 activity was calculated by dividing the activity value by the corresponding protein accumulation. 1010 Data represent means \pm S.D. $(n=3)$.

1011

1012 Figure 4. N-terminal fusion of an acyl-CoA binding protein (ACBP) with CzDGAT1 and its

1013 N-terminal truncation mutants. A, Protein production level of the full-length CzDGAT1 
1014 (DGAT1 1-550), N-terminal truncated CzDGAT1 (DGAT1 (D1-550 $_{1}$ and DGAT1 $107-550$ ) and their 1015 corresponding ACBP fused proteins (ACBP-DGAT11-550, ACBP-DGAT181-550 and ACBP-

1016 DGAT1 107-550). The enzyme amount was semi-quantified by Image J software (Schneider et al.,

1017 2012). B, In vitro DGAT activity of the full-length, $\mathrm{N}$-terminal truncated and ACBP fused

1018 CzDGAT1 proteins. C, Normalized DGAT activity of the full-length, N-terminal truncated and

1019 ACBP fused CzDGAT1 proteins. Normalized activity was calculated by dividing the activity

1020 value by the corresponding protein accumulation. Data represent means \pm S.D. $(n=3)$.

1021

1022 Figure 5. N-terminal fusion of an acyl-CoA binding protein (ACBP) with CzDGAT1

1023 kinetically improves the enzyme. A-D, DGAT activities of the full-length CzDGAT1

1024 (DGAT1 1-550), N-terminal truncated CzDGAT1 (DGAT1 1 1-550) and their corresponding ACBP 1025 fused proteins (ACBP-DGAT1 1-550 and ACBP-DGAT1 ${ }_{81-550}$ ) at oleoyl-CoA concentration from $10260.1-7.5$ or $10 \mu \mathrm{M}$. Data were fitted to the allosteric sigmoidal equation using GraphPad Prism. 1027 Data represent means \pm S.D. $(n=3)$. ACBP-fused DGAT1. B, Triacylglycerol (TAG) content in transiently transformed $N$. benthamiana leaves. All cDNAs were constitutively expressed under the CaMV $35 S$ promoter

1033 with the co-expression of the $p 19$ vector and Arabidopsis thaliana Wrinkled1 (AtWRI1). C, Fatty 1034 acid composition of TAG in in transiently transformed $N$. benthamiana leaves. D, Subcellular 1035 localization of ACBP-fused DGAT1 in $N$. benthamiana leaf cells. Venus-tagged DGAT1 or 1036 ACBP-DGAT1 was co-localized with C-terminal SCFP3A tagged Arabidopsis thaliana 1037 glycerol-3-phosphate acyltransferase (AtGPAT9), a known endoplasmic reticulum (ER) 1038 localized protein. Scale bars represent $20 \mu \mathrm{m}$. E, Fluorescence intensity of ACBP-fused DGAT1. $1039 N$. benthamiana leaves transiently expressing Venus-DGAT1 or Venus-ACBP-DGAT1 and the $1040 p 19$ vector were used to quantify the fluorescent intensity. F, Western-blot immunodetection 1041 detection against V5 tagged ACBP-fused DGAT1 or DGAT1 on $N$. benthamiana leaf proteins.

1042 Coomassie Blue staining of duplicate leaf protein samples separated on SDS-PAGE gel is shown 1043 as a loading control. G, Relative protein accumulation levels of ACBP-fused DGAT1 or DGAT1 
1044 in N. benthamiana leaf cells based on western blot analysis. The enzyme amount was semi1045 quantified by Image J software (Schneider et al., 2012). C16:0, Palmitic acid; C18:0, Stearic acid;

1046 C18:1, Oleic acid; C18:2, Linoleic acid; C18:3, $\alpha$-linolenic acid; p19, p19 + AtWRI1; DGAT1

1047 550, p19 + AtWRI1 + CzDGAT1 1-550; ACBP-DGAT1 1-550, p19 + AtWRI1 + ACBP fused

1048 CzDGAT1 1-550; ACBP+DGAT1 1-550, p19 + AtWRI1 + ACBP + CzDGAT1 1-550; DGAT181-550,

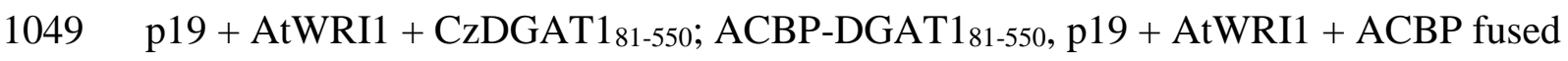

1050 CzDGAT181-550; ACBP+DGAT181-550, p19 + AtWRI1 + ACBP + CzDGAT1 81-550. Data represent

1051 means \pm S.D. For $A$ and $G, n=3$; for $B$ and $C, n=7$; for $E, n=4$. The asterisk and pound sign

1052 indicate $P<0.05$ as determined by paired one-tailed T-test. 


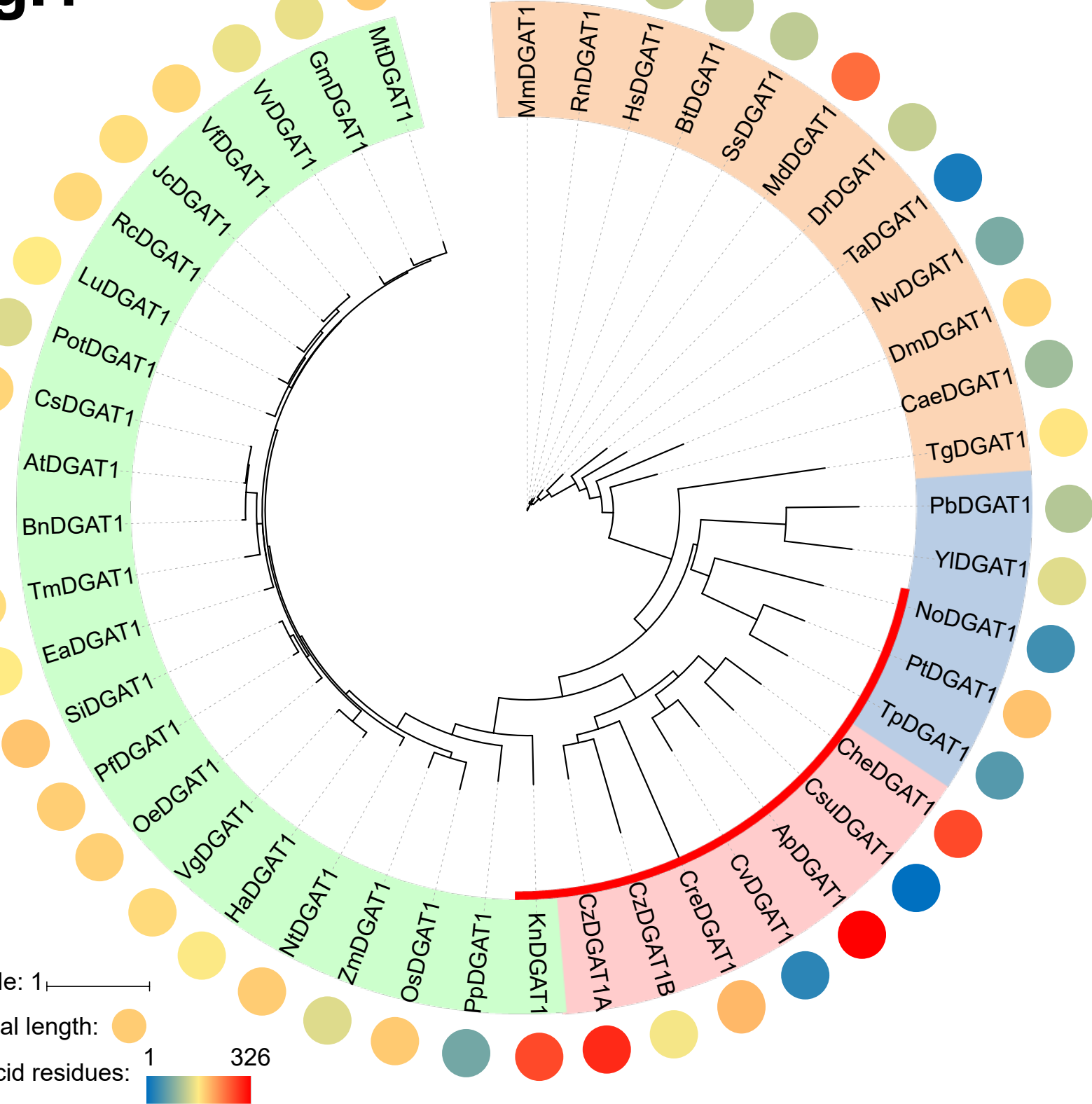

Tree scale: $1 \vdash$

N-terminal length: Amino acid residues:

326 

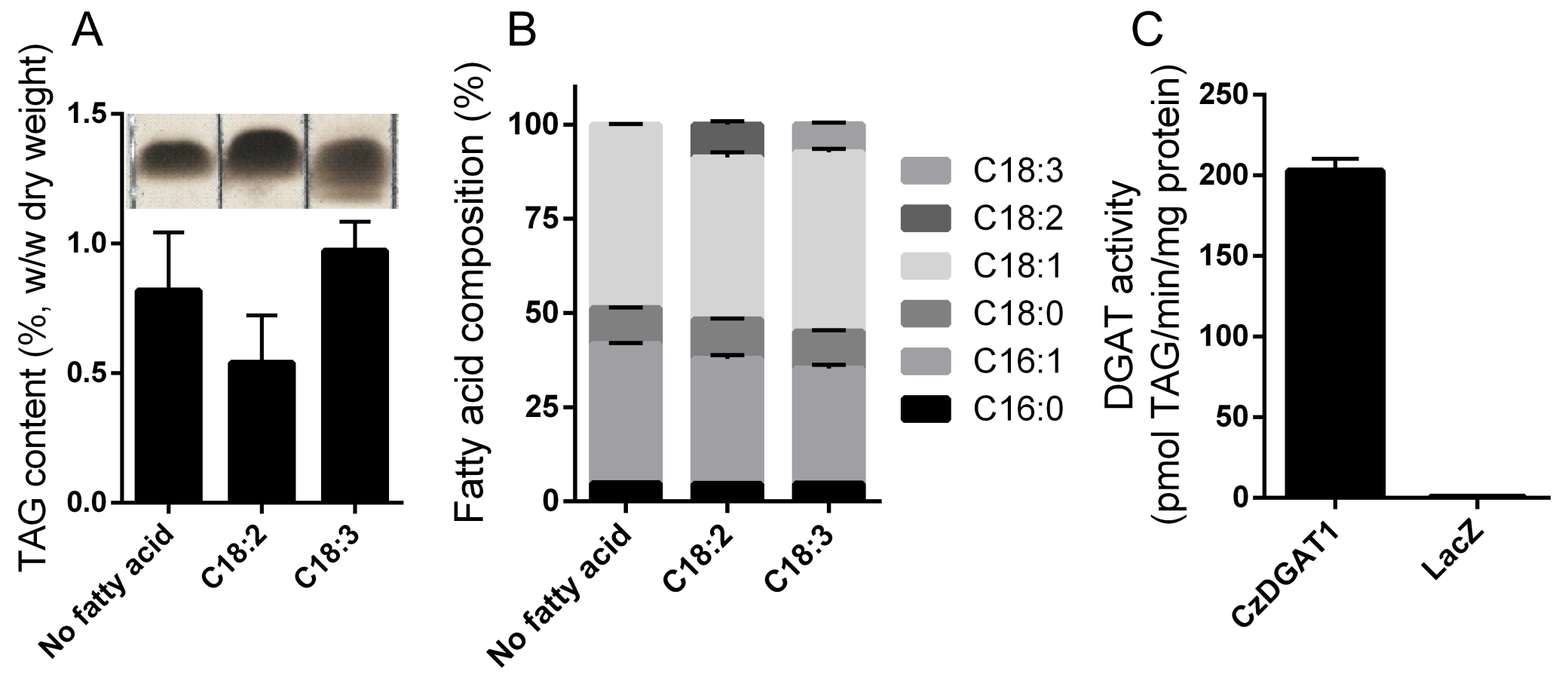

D

1

10

20

30

40

50

60

AtDGAT1 MALIDSAGVTTVTENGGGEFVDLDRLRRRK SRS DS SNG LLLSGS DNN SPS DDV GAP ADVRDR IDSVVIND BnDGAT1 MEILDSGGVTMP TENGGA---DLDTLRHRK PRS DS SNG LLP DSV --------TVSDADVRDRVDSAVE D MmDGAT1

CzDGAT1

CVDGAT1

MGDRGGA-- GSSRRRRTGSRVSVQGG---n

NoDGAT1 MEGARITTRPSPVTVAADELPETPFSSVSYDTSADADAIDSHQDQDVIS

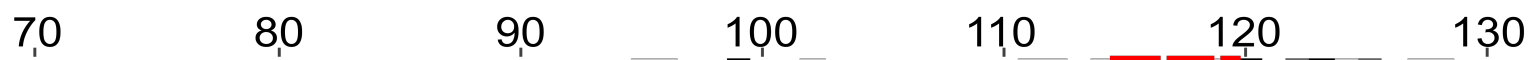

AtDGAT1 DAQGTANLAGDNNGGGDNNGGGRGGGEGRGNADA TFTYRP SVPAHRRARESP ISSDAI FKQSH

BnDGAT1 TQGKANIAGENEIRES-------GGEAGGNVDVRYTYRP SVPAHRRVRESP ISSSDAIFKQSH

MmDGAT1 DLGAGGDAPAPAPAPAHTRDKDGRTSVGDGYWDLRC----

CZDGAT1 RRKRAKSAWNR LIRTET LINEAT PRGQKTGPMIGSS------PLSYVTRD|SYISIQTVWTEKH

CvDGAT1

NoDGAT1

-Disordered state

MS MHK LTRPSNLSIEYP SRDYT

MAPRASIISSGRIRLINQ

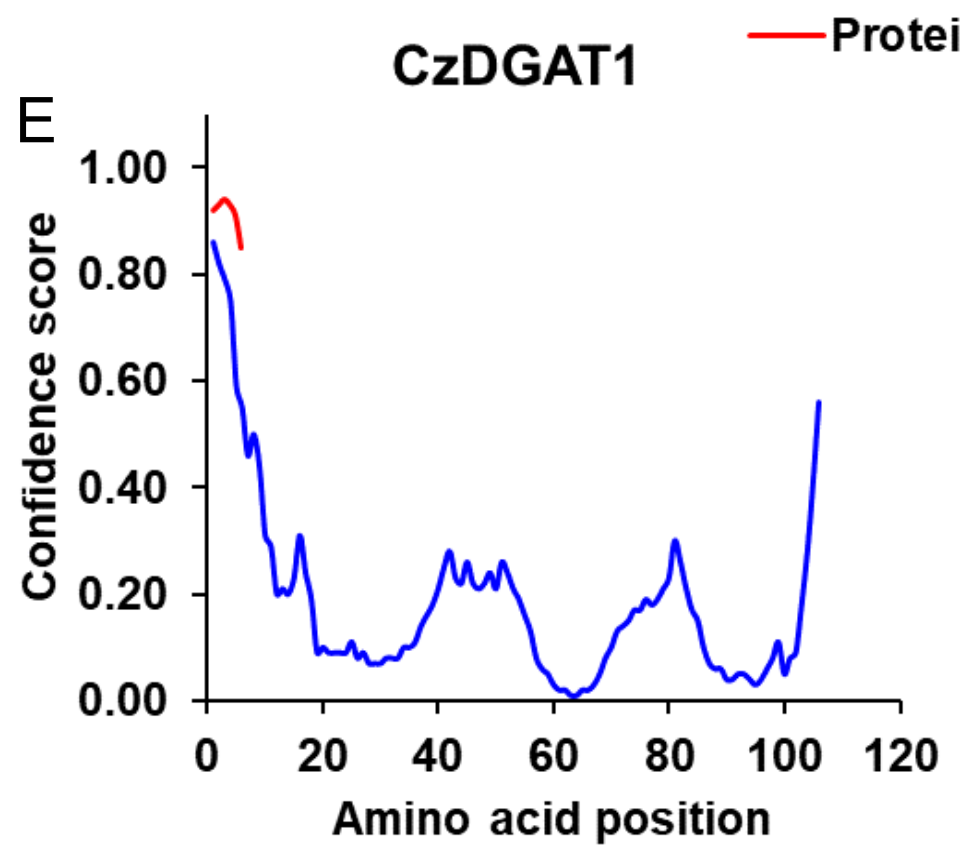

BnDGAT1

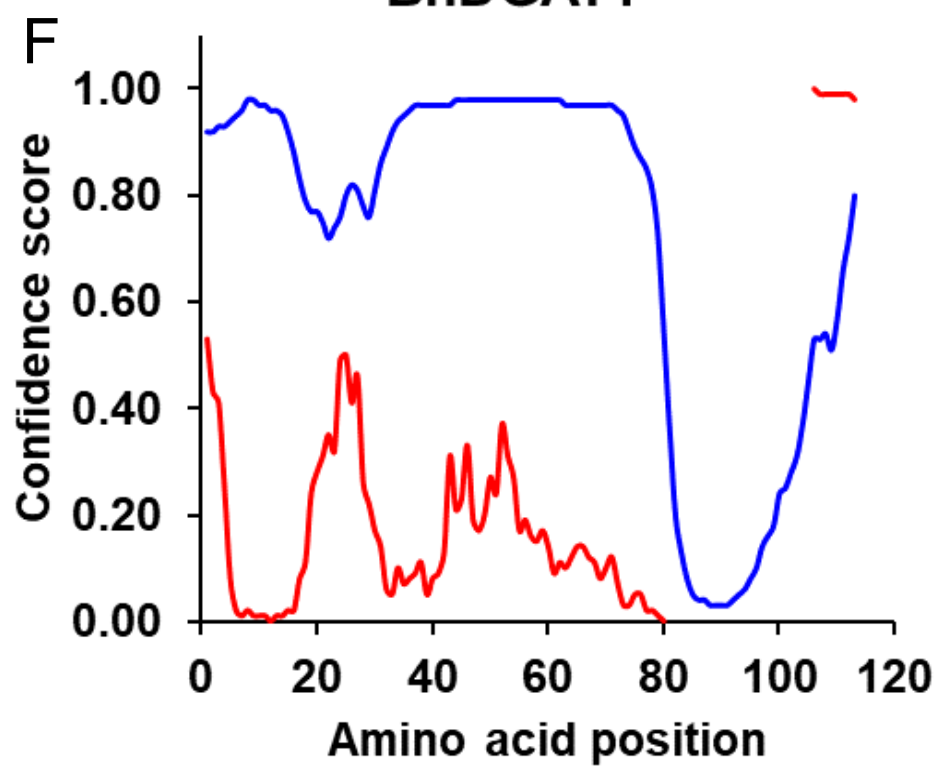




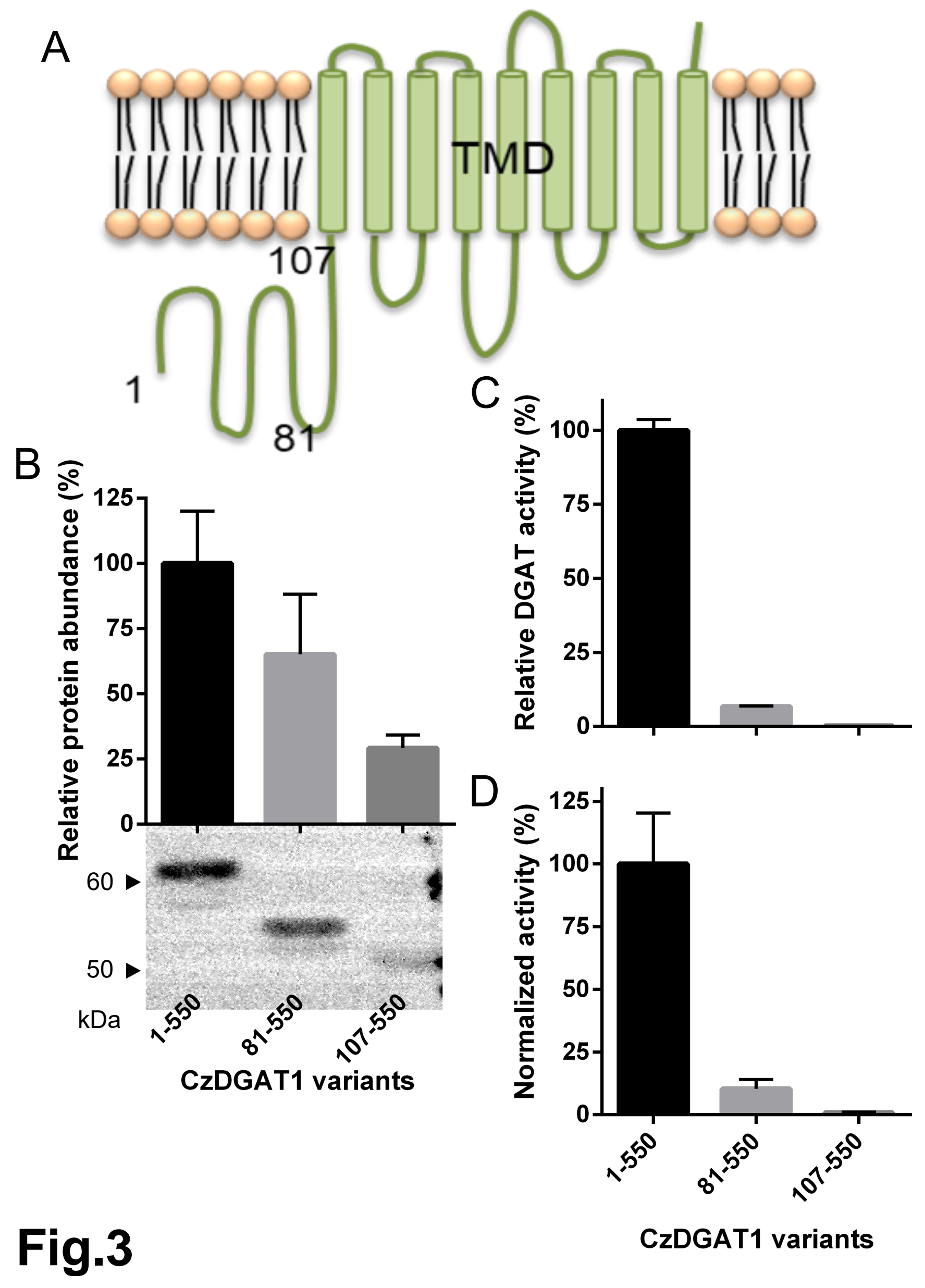



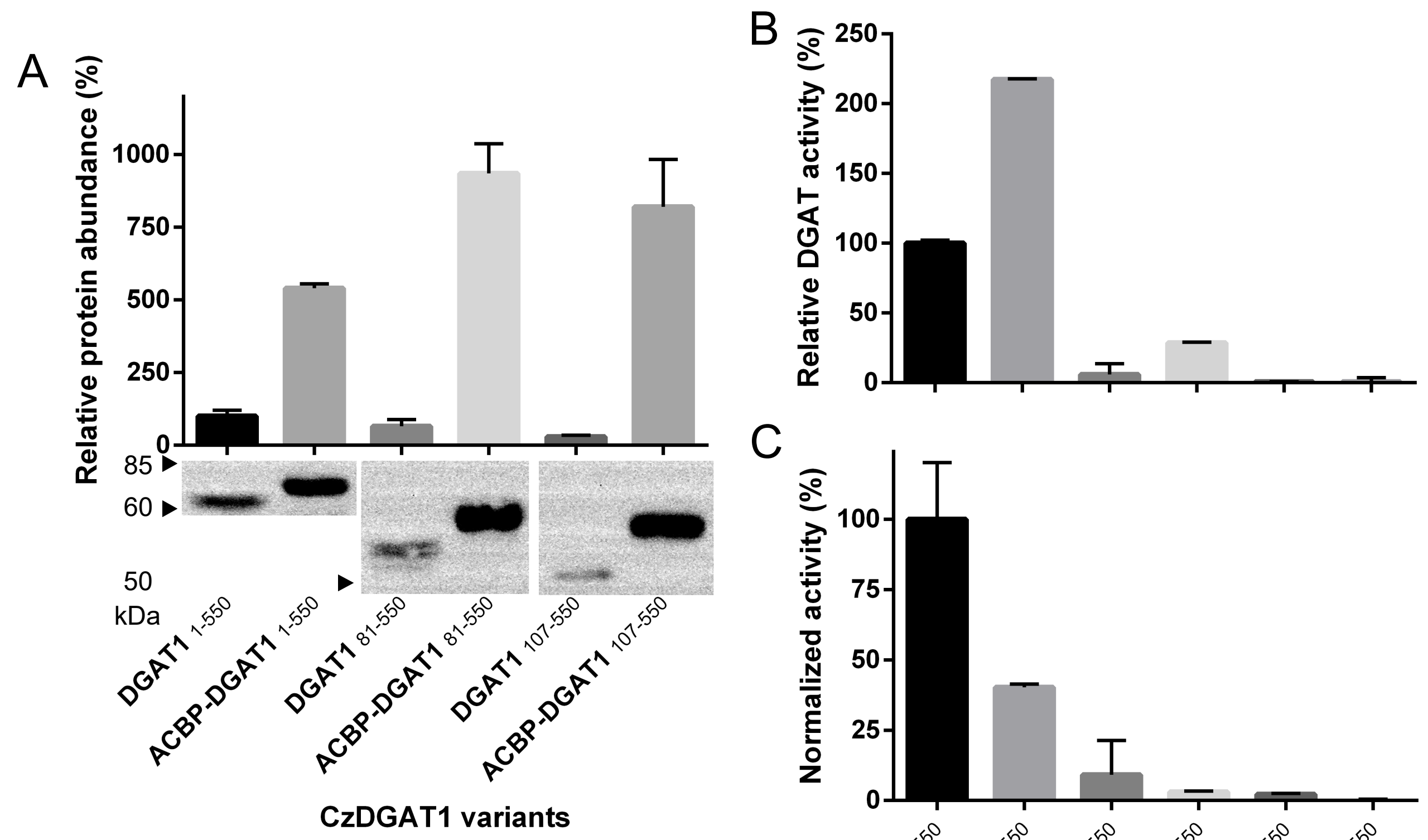

C

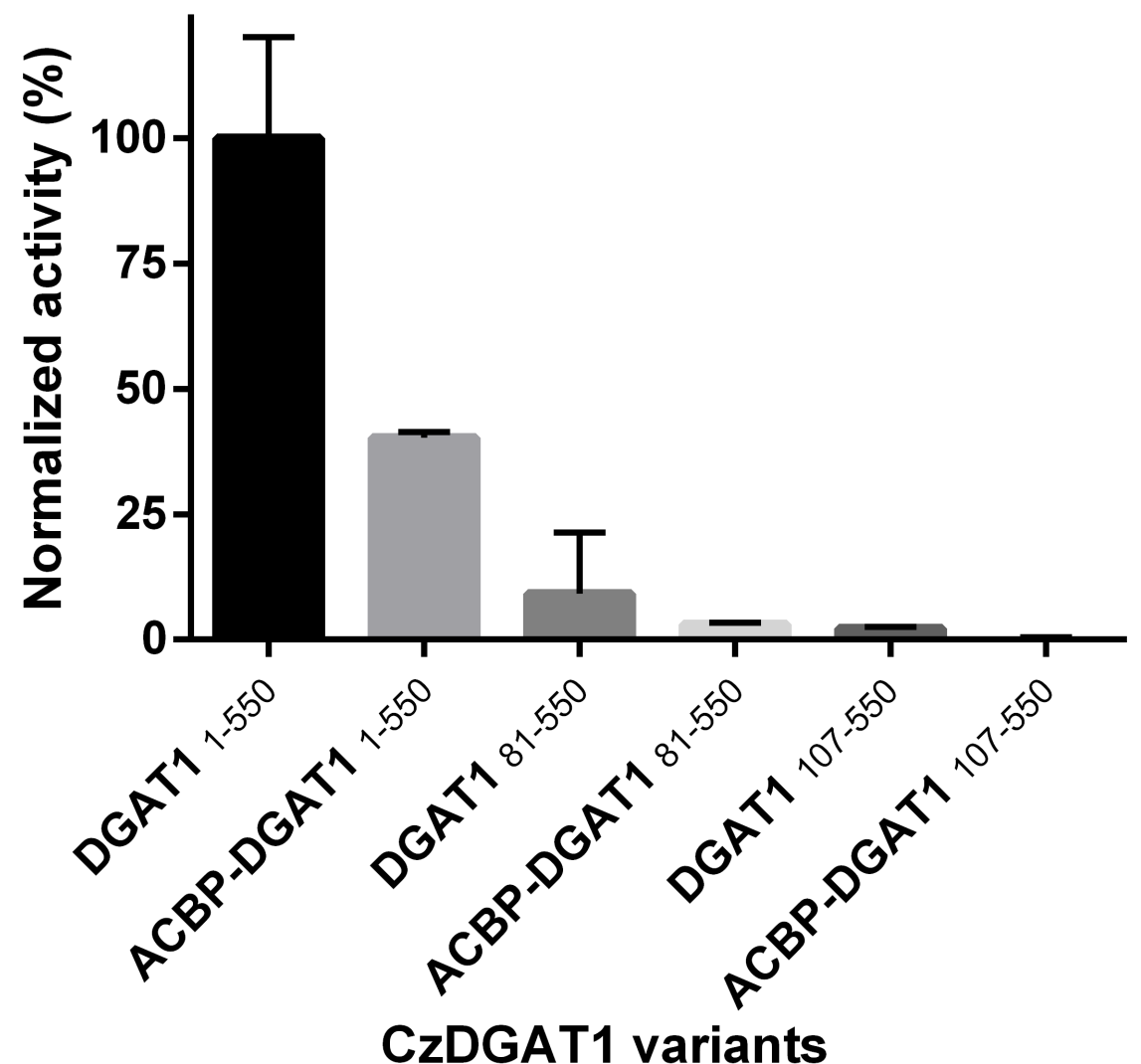

Fig.4 
Fig.5

A

CzDGAT1 $_{1-550}$

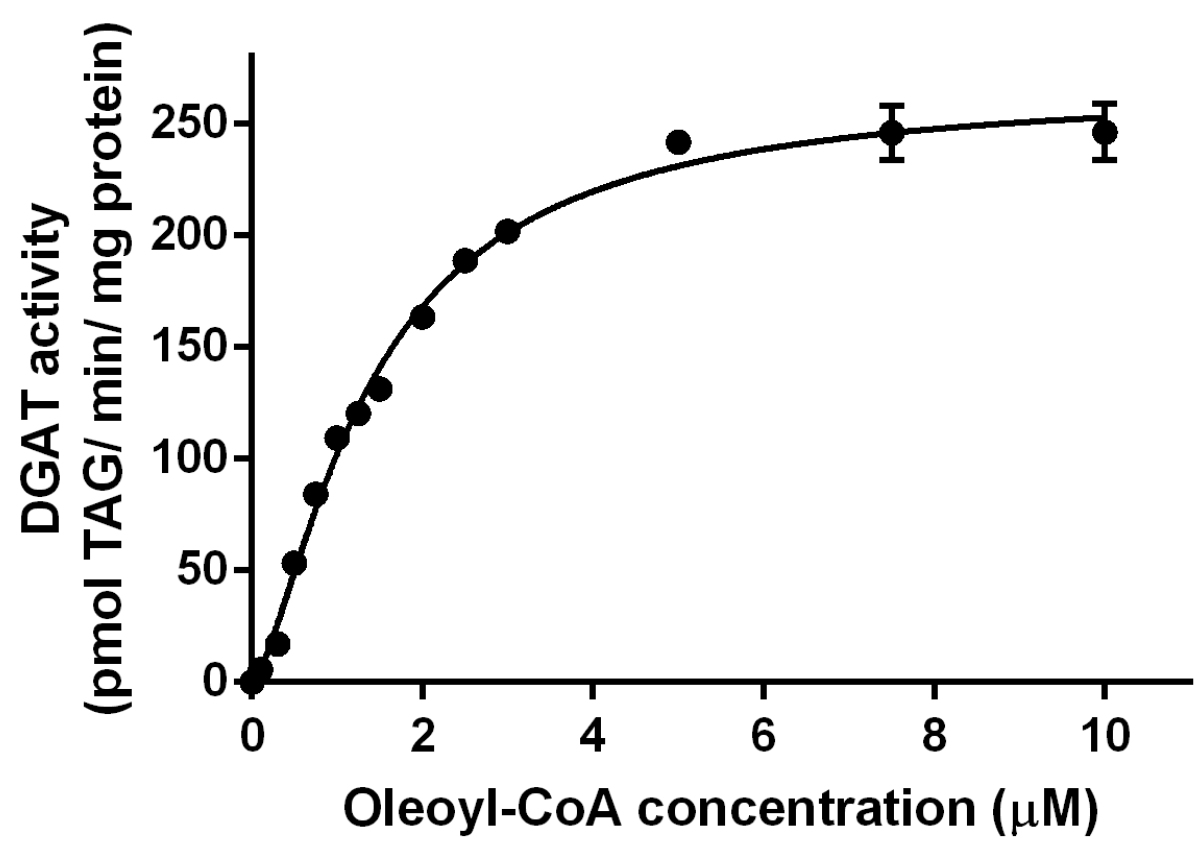

C

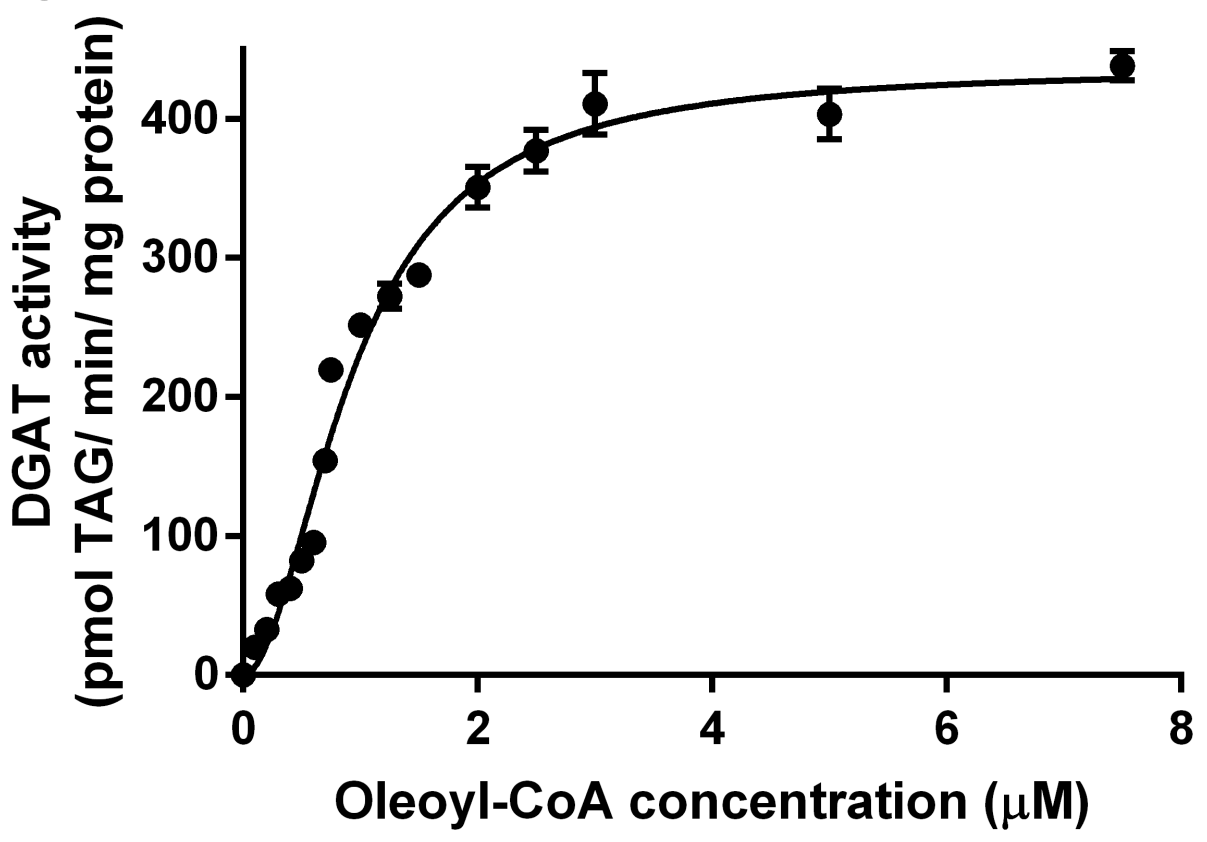

B CzDGAT1 11-550

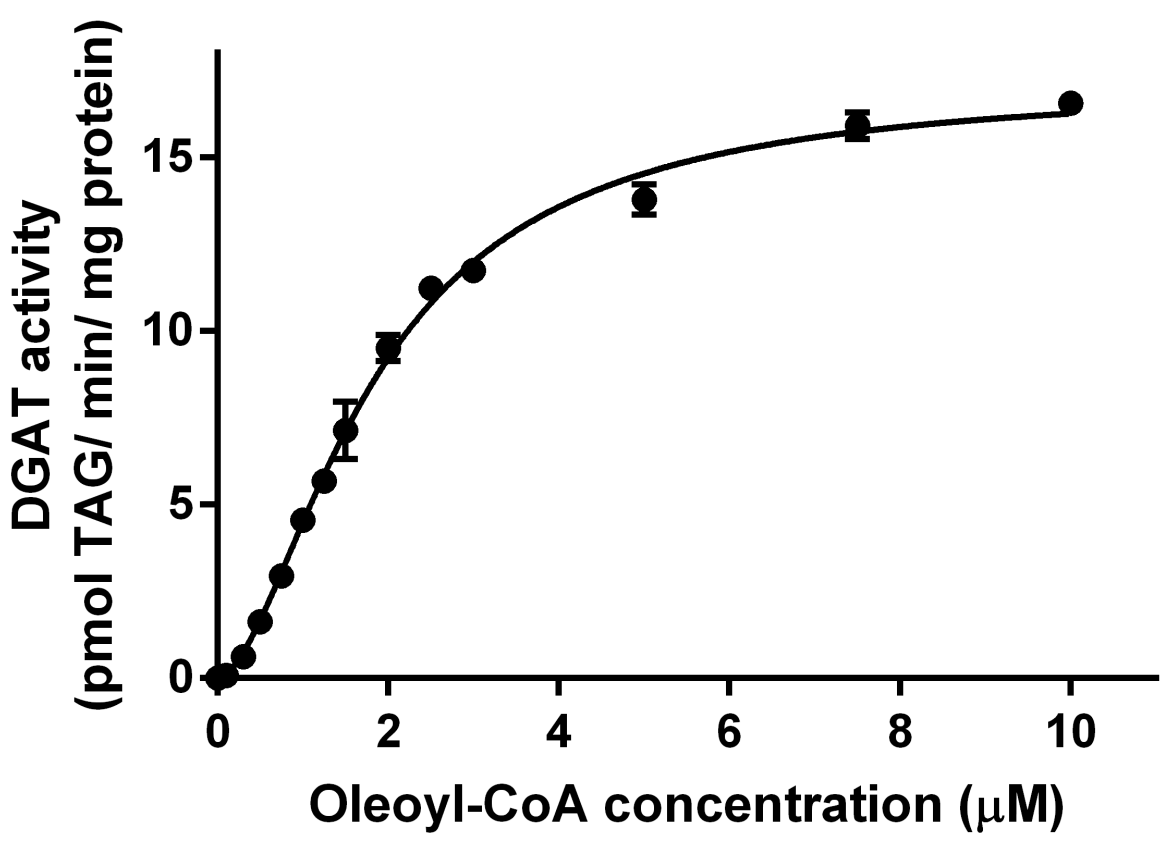

D

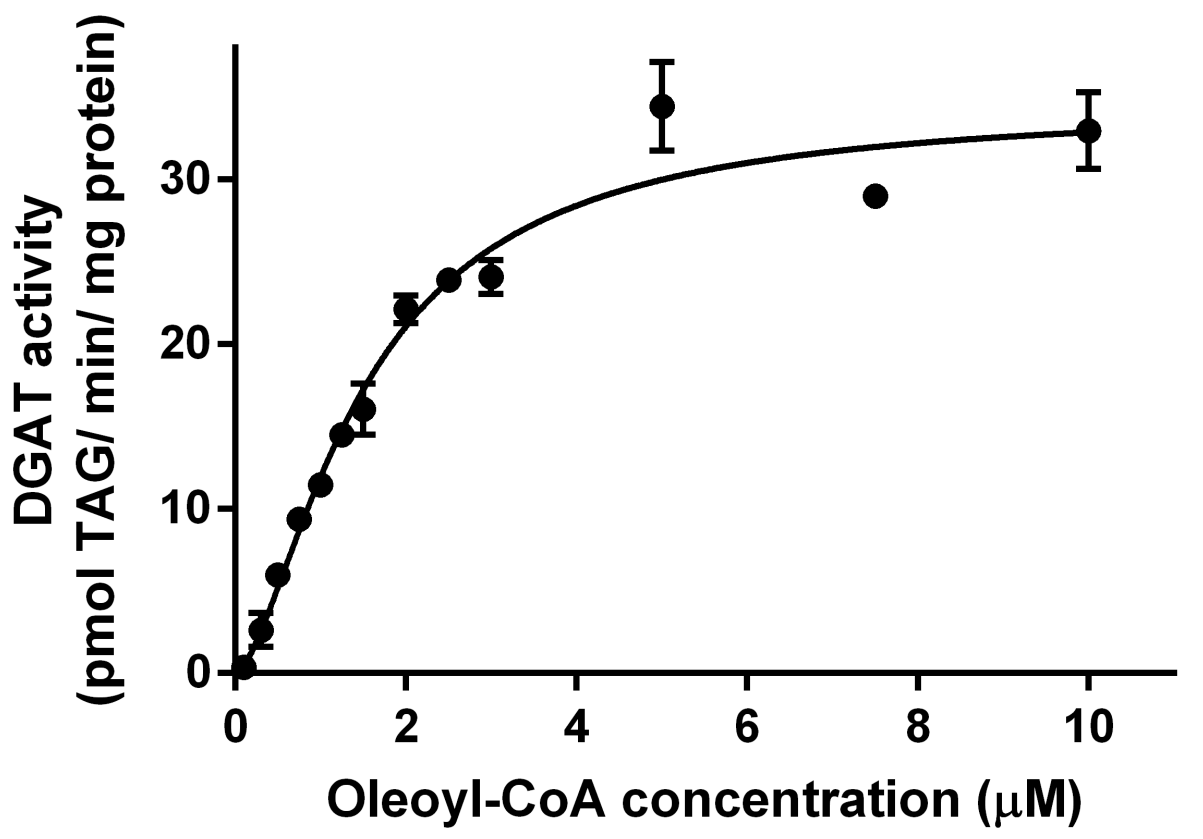


Fig.6

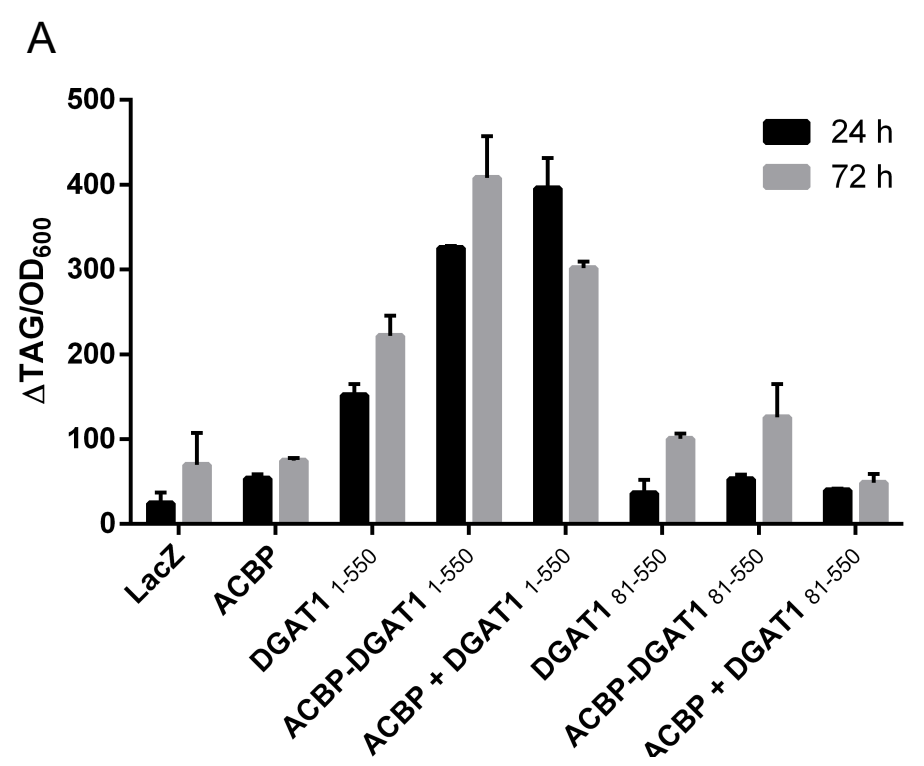

D
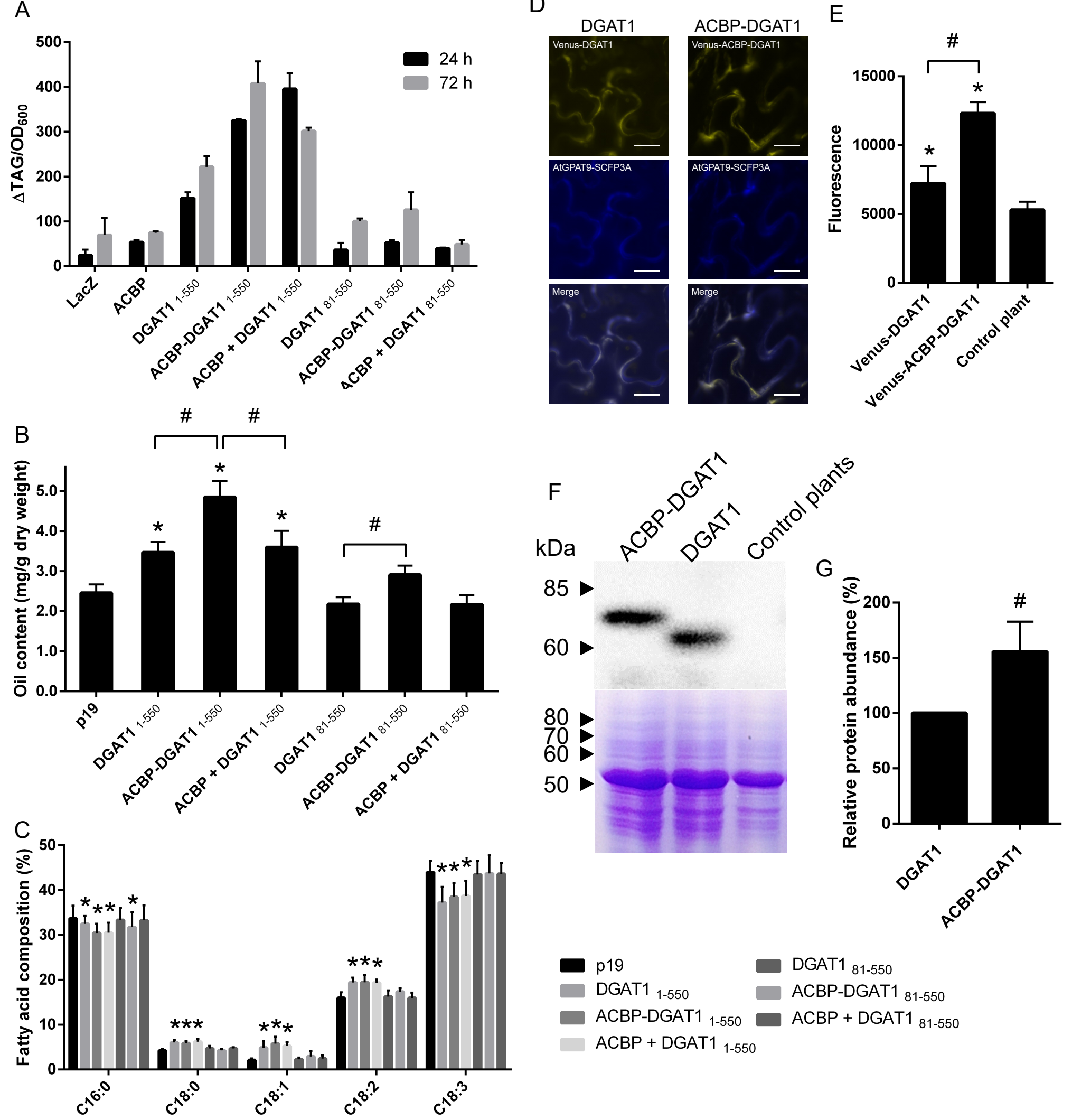

DGAT1 1 1-550

DGAT1 $81-550$

ACBP-DGAT1 ${ }_{1-550}$

ACBP-DGAT1 $81-550$

ACBP + DGAT1 ${ }_{1-550}$

ACBP + DGAT1 $81-550$ 